\title{
An Improved Near-infrared Spectrum of the Archetype Y Dwarf WISEP J182831.08+265037.8
}

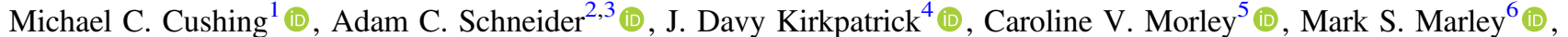 \\ Christopher R. Gelino ${ }^{4}$, Gregory N. Mace ${ }^{7}$, Edward L. Wright $^{8}{ }^{(1)}$, Peter R. Eisenhardt ${ }^{9}$, Michael F. Skrutskie ${ }^{10}$ (I) , and \\ Kenneth A. Marsh ${ }^{4}$ (1) \\ ${ }^{1}$ Ritter Astrophysical Research Center, Department of Physics and Astronomy, University of Toledo, 2801 W. Bancroft Street, Toledo, OH 43606, USA \\ michael.cushing@utoledo.edu \\ ${ }^{2}$ US Naval Observatory, Flagstaff Station, P.O. Box 1149, Flagstaff, AZ 86002, USA \\ ${ }^{3}$ Department of Physics and Astronomy, George Mason University, MS3F3, 4400 University Drive, Fairfax, VA 22030, USA \\ ${ }^{4}$ IPAC, Mail Code 100-22, Caltech, 1200 E. California Boulevard, Pasadena, CA 91125, USA \\ Department of Astronomy, University of Texas at Austin, Austin, TX, USA \\ ${ }^{6}$ Lunar and Planetary Laboratory, University of Arizona, 1629 E. University Boulevard, Tucson, AZ 85721, USA \\ ${ }^{7}$ McDonald Observatory and Department of Astronomy, University of Texas at Austin, 2515 Speedway, Stop C1400, Austin, TX 78712-1205, USA \\ ${ }^{8}$ Department of Physics and Astronomy, UCLA, 430 Portola Plaza, Box 951547, Los Angeles, CA 90095-1547, USA \\ 9 Jet Propulsion Laboratory, California Institute of Technology, 4800 Oak Grove Drive, Pasadena, CA 91109, USA \\ ${ }^{10}$ Department of Astronomy, University of Virginia, Charlottesville, VA 22904, USA \\ Received 2021 January 12; revised 2021 June 24; accepted 2021 June 28; published 2021 October 8
}

\begin{abstract}
We present a Hubble Space Telescope/Wide-Field Camera 3 near-infrared spectrum of the archetype Y dwarf WISEP 182831.08+265037.8. The spectrum covers the 0.9-1.7 $\mu \mathrm{m}$ wavelength range at a resolving power of $\lambda /$ $\Delta \lambda \approx 180$ and is a significant improvement over the previously published spectrum because it covers a broader wavelength range and is uncontaminated by light from a background star. The spectrum is unique for a cool brown dwarf in that the flux peaks in the $Y, J$, and $H$ bands are of near equal intensity in units of $f_{\lambda}$. We fail to detect any absorption bands of $\mathrm{NH}_{3}$ in the spectrum, in contrast to the predictions of chemical equilibrium models, but tentatively identify $\mathrm{CH}_{4}$ as the carrier of an unknown absorption feature centered at $1.015 \mu \mathrm{m}$. Using previously published ground- and spaced-based photometry, and using a Rayleigh-Jeans tail to account for flux emerging longward of $4.5 \mu \mathrm{m}$, we compute a bolometric luminosity of $\log \left(L_{\mathrm{bol}} / \mathcal{L}_{\odot}^{N}\right)=-6.50 \pm 0.02$, which is significantly lower than previously published results. Finally, we compare the spectrum and photometry to two sets of atmospheric models and find that the best overall match to the observed properties of WISE $1828+2650$ is a $\sim 1$ Gyr old binary composed of two $T_{\text {eff }} \sim 325 \mathrm{~K}, \sim 5 M_{\text {Jup }}$ brown dwarfs with subsolar [C/O] ratios.
\end{abstract}

Unified Astronomy Thesaurus concepts: Near infrared astronomy (1093); Y dwarfs (1827); Spectroscopy (1558); Brown dwarfs (185)

Supporting material: data behind figure

\section{Introduction}

The search for cool brown dwarfs with effective temperatures $\left(T_{\text {eff }}\right)$ less than $700 \mathrm{~K}$ (approximately a spectral type of T8) was one of the driving science goals of wide-area, redoptical, and infrared surveys such as the United Kingdom Infrared Telescope Infrared Deep Sky Survey (UKIDSS; Lawrence et al. 2007), the Canada France Brown Dwarf Survey (CFBDS; Delorme et al. 2008b) and its counterpart in the near-infrared CFBDSIR (Delorme et al. 2010), and the Wide-field Infrared Survey Explorer (WISE; Wright et al. 2010). With masses less than $\sim 20 M_{\text {Jup }}$ at the typical age of the ultracool dwarf field population of $3 \mathrm{Gyr}$ (Dahn et al. 2002), such cool brown dwarfs are most likely the least massive products of star formation (e.g., Kirkpatrick et al. 2021).

While such cool brown dwarfs have been discovered using each of these surveys (e.g., Burningham et al. 2008; Delorme et al. 2008a, 2010; Cushing et al. 2011; Kirkpatrick et al. 2011; Liu et al. 2011) the vast majority of brown dwarfs with effective temperatures less than $500 \mathrm{~K}$ have been discovered using WISE data. The near-infrared spectrum of one such brown dwarf, WISEP J182831.08+265037.8 (hereafter WISE $1828+2650$ ), was distinct enough from that of the late-type $\mathrm{T}$ dwarfs that Cushing et al. (2011) identified it has the archetype of the new Y spectral class. They originally assigned it a spectral type of $>\mathrm{Y} 0$ but its type was later revised to $\geqslant \mathrm{Y} 2$ after the discovery of the Y1 dwarf WISE $035000.32-565830.2$ by Kirkpatrick et al. (2012). At the time of its discovery, WISE $1828+2650$ had the reddest near- to mid-infrared color of any brown dwarf known at $J-W 2=9.29 \pm 0.35$ (mag). Atmospheric models indicated such a color corresponded to an effective temperature of less than $\sim 300 \mathrm{~K}$.

Once its parallax was measured (Beichman et al. 2013; Dupuy \& Kraus 2013), it became clear that WISE $1828+2650$ was overluminous in nearly all color-magnitude and color spectral type diagrams (Kirkpatrick et al. 2012; Beichman et al. 2013; Dupuy \& Kraus 2013; Leggett et al. 2013). Several reasons for this over-luminosity were suggested including misclassification (Kirkpatrick et al. 2012), a brightening in the spectral sequence due to an unknown physical mechanism (Kirkpatrick et al. 2012; Cushing 2014), and unresolved binarity (Beichman et al. 2013; Leggett et al. 2013). The subsequent measurement of the parallaxes for the even redder and cooler brown dwarfs WD 0806-661B (Luhman et al. 2011) and WISE J085510.83-071442.5 (hereafter WISE 0855 -0714; Luhman 2014) strongly suggests binarity is the cause of WISE $1828+2650$ 's over-luminosity. This is because these two objects appear to fall in line with the other Y dwarfs in 
color-magnitude diagrams (Faherty et al. 2014; Tinney et al. 2014; Leggett et al. 2015; Schneider et al. 2016). Subsequent discoveries of brown dwarfs with similar effective temperatures like CWISEP J193518.59-154620.3 (Marocco et al. 2019), CWISEP J144606.62-231717.8 (Marocco et al. 2020; Meisner et al. 2020), and WISEA J083011.95+283716.0 (Bardalez Gagliuffi et al. 2020) have only strengthened the case for binarity, but WISE $1828+2650$ remains stubbornly unresolved in both Hubble Space Telescope (HST) and ground-based adaptive optics imaging (Cushing et al. 2011; Beichman et al. 2013). However, given the poor quality of model atmosphere fits to the near-to-mid-infrared spectral energy distribution of Y dwarfs (e.g., Schneider et al. 2015; Luhman \& Esplin 2016; Leggett et al. 2017), missing or incomplete opacity sources and chemistry cannot be ruled out.

The spectrum of WISE $1828+2650$ presented by Cushing et al. (2011) was obtained with the G141 grism in the Wide Field Camera 3 (WFC3; Kimble et al. 2008) on board the HST and covered $1.1-1.7 \mu \mathrm{m}$ at a resolving power of $R \equiv \lambda /$ $\Delta \lambda \approx 130$. However because WFC3 is slitless, extreme care must be taken in order to avoid contamination by light from stars nearby on the sky. At the time of the observations, we had yet to perfect our methodology for roll angle selection, and so the resulting spectrum was contaminated by light from such a star. In this paper, we present an improved, contamination-free HST/WFC3 spectrum that covers an even larger wavelength range of $0.9-1.7 \mu \mathrm{m}$.

In Section 2, we describe the acquisition and reduction of the spectrum. In Section 3, we discuss the characteristics of this unique spectrum in detail, revisit the spectral classification of WISE $1828+2650$ based on our new spectrum, and construct a spectral energy distribution covering $0.9-4.5 \mu \mathrm{m}$ using previously published photometry. Section 4 presents our estimates for the bolometric luminosity and effective temperature of WISE $1828+2650$. The latter quantity was determined using both evolutionary models and two different sets of atmospheric models. Finally in Section 5, we discuss the model spectra fits to the data and the implications for the properties of WISE $1828+2650$.

\section{Observations and Data Reduction}

WISE $1828+2650$ was observed with the WFC3 on board HST as a part of a Cycle 20 program to obtain grism spectroscopy of late-type $\mathrm{T}$ and $\mathrm{Y}$ dwarf candidates (GO12970, PI=Cushing). The WFC3 uses a $1024 \times 1024 \mathrm{HgCdTe}$ detector with a plate scale of 0 ". 13 pixel $^{-1}$, which results in a field of view of $123^{\prime \prime} \times 126^{\prime \prime}$. WISE $1828+2650$ was observed with the G102 grism, which covers the $0.8-1.15 \mu \mathrm{m}$ wavelength range at a resolving power of $R \sim 210$, and the G141 grism, which covers the $1.075-1.70 \mu \mathrm{m}$ wavelength range at a resolving power of $R \sim 130$. Direct images through the F105W $\left(\lambda_{\text {pivot }}=1.0552 \mu \mathrm{m}, \Delta \lambda=0.265 \mu \mathrm{m}\right)$ or F125W $\left(\lambda_{\text {pivot }}=1.2486 \mu \mathrm{m}, \Delta \lambda=0.2845 \mu \mathrm{m}\right)$ filter were also obtained before the G102 and G141 grism images, respectively. A $\log$ of the observations is given in Table 1. Both the spectroscopic and photometric observations were reduced as described in Schneider et al. (2015); the resulting G102+G141 spectrum is shown in Figure 1 while the F105W and F125W magnitudes are given in Table 2 . There is a $2 \%$ uncertainty in the absolute flux calibration of the spectrum (Kuntschner et al. $2011)$ and the signal-to-noise ratio $(\mathrm{S} / \mathrm{N})$ of the spectrum at the peaks of the $Y, J$, and $H$ bands $(1.05,1.27$, and $1.6 \mu \mathrm{m})$ ranges
Table 1

Log of HST/WFC3 Observations

\begin{tabular}{lcc}
\hline \hline UT Date & Grism/Filter & Total Integration Time (s) \\
\hline 2013 Apr 22 & F105 & 509 \\
2013 Apr 22 & G102 & 7210 \\
2013 May 6 & F125 & 559 \\
2013 May 6 & G141 & 7210 \\
2013 May 8 & F105 & 509 \\
2013 May 8 & G102 & 7210 \\
2013 Jun 21 & F105 & 509 \\
2013 Jun 21 & G102 & 7210 \\
2013 Jun 25 & F105 & 509 \\
2013 Jun 25 & G102 & 7210 \\
2013 Aug 14 & F125 & 559 \\
2013 Aug 14 & G141 & 7210 \\
2013 Aug 17 & F105 & 509 \\
2013 Aug 17 & G102 & 7210 \\
\hline
\end{tabular}

from 15 to 20 . We also computed the F140W $\left(\lambda_{\text {pivot }}=\right.$ $1.3923 \mu \mathrm{m}, \Delta \lambda=0.384 \mu \mathrm{m})$ magnitude of WISE $1828+2650$ using the direct images obtained with the original G141 grism spectrum, which is also given in Table 2 .

\section{The Spectrum}

The spectrum exhibits deep absorption bands of $\mathrm{CH}_{4}$ and $\mathrm{H}_{2} \mathrm{O}$ typical of late-type $\mathrm{T}$ dwarf spectra (e.g., Burgasser et al. 2006), but the heights of the $Y$-, $J$-, and $H$-band peaks are approximately equal in units of $f_{\lambda}$. This atypical characteristic prompted Cushing et al. (2011) to identify WISE 1828+2650 as the first Y dwarf.

We tentatively identify the $3\left(\nu_{1}, \nu_{3}\right)$ band head of $\mathrm{H}_{2} \mathrm{O}$ at $0.925 \mu \mathrm{m}$ in the spectrum of WISE $1828+2650$. This band head is prominent in the spectra of the $\mathrm{T}$ dwarfs (Burgasser et al. 2003; Kirkpatrick et al. 2011) and has even been detected in the spectrum of the Y0 dwarf WISEPC J205628.90 +145953.3 (Leggett et al. 2013). However given the low $\mathrm{S} / \mathrm{N}$ at these wavelengths and the fact that the feature falls at the end of the spectrum, a higher $\mathrm{S} / \mathrm{N}$ spectrum will be required in order to confirm the band head is real and carried by $\mathrm{H}_{2} \mathrm{O}$.

Ammonia is also an important opacity source in the atmospheres of cool brown dwarfs because it becomes the dominant equilibrium chemistry nitrogen-bearing species $\left(A\left(\mathrm{~N}_{2}\right) / A\left(\mathrm{NH}_{3}\right)<1\right)$ at $T \lesssim 700 \mathrm{~K}$ (Lodders \& Fegley 2002). The $\nu_{2}$ fundamental band is found in the spectra of T dwarfs at $10.5 \mu \mathrm{m}$ (Roellig et al. 2004; Cushing et al. 2006), but the overtone and combination bands in the near-infrared (1.03, $1.21,1.31,1.51,1.66,1.98$, and $2.26 \mu \mathrm{m}$ ) have defied clear detection because they are intrinsically weaker than the fundamental bands and often overlap in wavelength with strong $\mathrm{H}_{2} \mathrm{O}$ and $\mathrm{CH}_{4}$ overtone and combination bands.

It has long been suggested that the emergence of these bands could trigger the creation of a new spectral class (Burrows et al. 2003; Leggett et al. 2007; Kirkpatrick 2008), and indeed Cushing et al. (2011) set the transition between the $\mathrm{T}$ and $\mathrm{Y}$ dwarfs where the Delorme et al. (2008a) $\mathrm{NH}_{3}-H$ spectral index, which measures the strength of the $\mathrm{NH}_{3}$ absorption band on the blue side of the $H$-band peak, indicated a significant increase in absorption. Bochanski et al. (2011) identified weak $\mathrm{NH}_{3}$ features in a moderate resolution $(R=6000)$ spectrum of the T9 dwarf UGPS 0722-05 across the entire near-infrared wavelength range, but many of the identifications were later 


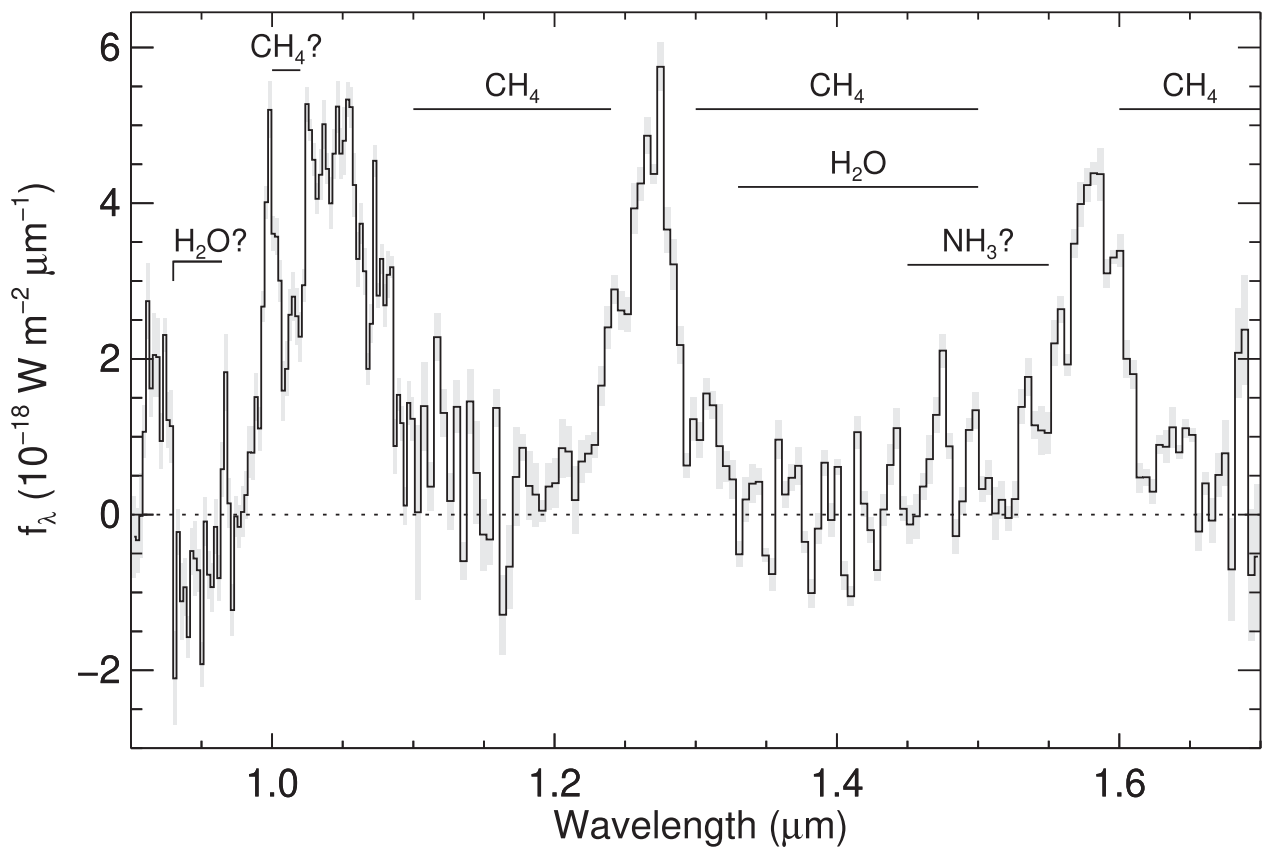

Figure 1. New G102+G141 HST/WFC3 spectrum of WISE 1828+2650. Prominent $\mathrm{H}_{2} \mathrm{O}$ and $\mathrm{CH}_{4}$ absorption bands are indicated along with potential absorption from the $\nu_{2}+\nu_{3} \mathrm{NH}_{3}$ band from 1.45 to $1.55 \mu \mathrm{m}$ and tentative $3\left(\nu_{1}, \nu_{3}\right) \mathrm{H}_{2} \mathrm{O}$ band head at $0.925 \mu \mathrm{m}$. The spectrum is available as the data behind the Figure.

(The data used to create this figure are available.)

Table 2

Summary of WISE $1828+2650$ Photometry

\begin{tabular}{lcc}
\hline \hline Parameter & Value & Reference \\
\hline F105W & $23.95 \pm 0.09 \mathrm{mag}$ & this work \\
F125W & $23.82 \pm 0.12 \mathrm{mag}$ & this work \\
F140W & $23.15 \pm 0.14 \mathrm{mag}$ & this work \\
$z$ (AB) & $>24.46 \mathrm{mag}$ & Lodieu et al. (2013) \\
$Y$ & $23.20 \pm 0.17 \mathrm{mag}$ & Leggett et al. (2013) \\
$J$ & $23.48 \pm 0.23 \mathrm{mag}$ & Leggett et al. (2013) \\
$H$ & $22.45 \pm 0.08 \mathrm{mag}$ & Beichman et al. (2013) \\
$K$ & $23.48 \pm 0.36 \mathrm{mag}$ & Leggett et al. (2013) \\
{$[3.6]$} & $16.915 \pm 0.020 \mathrm{mag}$ & Kirkpatrick et al. (2019) \\
[4.5] & $14.321 \pm 0.020 \mathrm{mag}$ & Kirkpatrick et al. (2019) \\
AllWISE $W 1$ & $>18.248 \mathrm{mag}^{\mathrm{a}}$ & Cutri et al. (2013) \\
AllWISE $W 2$ & $14.353 \pm 0.045 \mathrm{mag}^{2}$ & Cutri et al. (2013) \\
AllWISE W3 & $12.444 \pm 0.338 \mathrm{mag}^{\mathrm{b}}$ & Cutri et al. (2013) \\
AllWISE $W 4$ & $>8.505 \mathrm{mag}^{\mathrm{a}}$ & Cutri et al. (2013) \\
CatWISE2020 W1 & $18.823 \pm 0.224 \mathrm{mag}^{\mathrm{c}}$ & (Marocco et al. 2021) \\
CatWISE2020 W2 & $14.393 \pm 0.016 \mathrm{mag}^{2}$ & (Marocco et al. 2021) \\
\hline
\end{tabular}

Notes.

a The flux measurement has $\mathrm{S} / \mathrm{N}<2$; the magnitude quoted is derived from the $95 \%$ confidence flux upper limit.

${ }^{\mathrm{b}} \mathrm{S} / \mathrm{N}=3.2$ with no clear source on the atlas tile.

${ }^{\mathrm{c}} \mathrm{S} / \mathrm{N}=2.9$ but a faint source is visible, and the proper motion of the source agrees with published results obtained with Spitzer.

called into question by Saumon et al. (2012). More recently, Zalesky et al. (2019) measured the abundances of $\mathrm{NH}_{3}$ in the atmospheres of eight $\mathrm{Y}$ dwarfs through a retrieval analysis of the Schneider et al. (2015) HST/WFC3 spectra, but no clear detection of $\mathrm{NH}_{3}$ absorption features was reported.

The peak absorption coefficients of the near-infrared $\mathrm{NH}_{3}$ bands decrease steadily as one moves to shorter wavelengths (Saumon et al. 2012), which suggests that all else being equal, longer wavelength bands would be easier to detect. While our spectrum does not extend to the $K$ band, a search for the 1.98 and $2.26 \mu \mathrm{m} \mathrm{NH}_{3}$ bands would be difficult since the $K$-band flux of late-type $\mathrm{T}$ and $\mathrm{Y}$ dwarfs is heavily suppressed due to strong $\mathrm{CH}_{4}$ and collision-induced $\mathrm{H}_{2}$ absorption. The $1.66 \mu \mathrm{m}$ band is centered beyond the wavelength limit of our spectrum but is also overwhelmed by $\mathrm{CH}_{4}$ absorption and so would nevertheless be difficult to identify. Visual inspection of the red side of the $H$-band peak shows no distinctive absorption feature due to the $1.51 \mu \mathrm{m} \mathrm{NH}_{3}$ band, although this could be a result of the low $\mathrm{S} / \mathrm{N}$ and/or low resolving power of the spectrum. We have computed the $\mathrm{NH}_{3}-H$ spectral index of Delorme et al. (2008a) for WISE $1828+2650$ and a sample of $\mathrm{T}$ and $\mathrm{Y}$ dwarfs; the results are shown in Figure 2. WISE $1828+2650$ deviates significantly from the trend set by the $\mathrm{T}$ and other $\mathrm{Y}$ dwarfs. If the index is actually measuring $\mathrm{NH}_{3}$ absorption, then the WISE $1828+2650$ value suggests that either $\mathrm{NH}_{3}$ is absent from the spectrum, or the index is no longer sensitive to $\mathrm{NH}_{3}$ at this spectral type. The 1.21 and $1.31 \mu \mathrm{m} \mathrm{NH} \mathrm{NH}_{3}$ bands are difficult to identify because they overlap in wavelength with the $\mathrm{CH}_{4}$ bands that give rise to the $J$-band emission peak and thus simply help to confine the emergent flux to an ever-narrowing wavelength range.

In contrast, the $1.03 \mu \mathrm{m} 2 \nu_{1}+2 \nu_{4}$ band is centered near the peak of the $Y$ band, which suggests it will be more easily identified. However, Schneider et al. (2015) found no evidence of this feature in HST/WFC3 spectra of other late-type T dwarfs and Y dwarfs. The first panel in the left column of Figure 3 shows the spectrum of WISE $1828+2650$ centered on the $Y$-band peak. There is a weak double-peaked absorption feature centered at $1.015 \mu \mathrm{m}$ that at first appears to be consistent with this $\mathrm{NH}_{3}$ band. However, also plotted in the second panel of the left column is the opacity cross-section spectrum of $\mathrm{NH}_{3}$ at $T=100 \mathrm{~K}$ and $P=0.1$ bar generated using the Yurchenko et al. (2011) $\mathrm{NH}_{3}$ line list. The position of the 


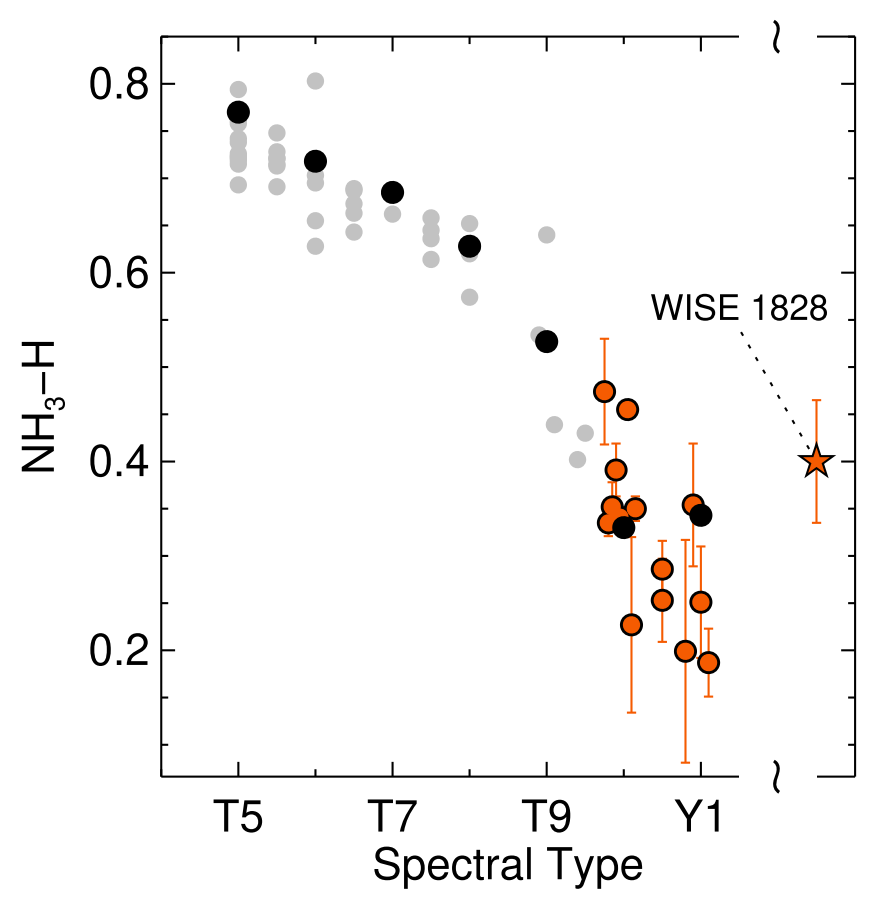

Figure 2. The Delorme et al. (2008a) $\mathrm{NH}_{3}-H$ spectral index as a function of spectral type. The black points are for the T5-Y1 spectral standards. The gray points were computed using SpeX/IRTF spectra from the SpeX Prism Library and the T dwarf spectra from Schneider et al. (2015) while the red points were computed using the Y dwarf spectra from Schneider et al. (2015) and from this work.

absorption feature in the spectrum of WISE $1828+2650$ clearly does not match the position of the $\mathrm{NH}_{3}$ band. Several possibilities present themselves:

1. The feature is real and is carried by $\mathrm{NH}_{3}$, but the Yurchenko et al. (2011) line list is in error. Laboratory observations of $\mathrm{NH}_{3}$ at similar temperatures and pressures by Cruikshank \& Binder (1969), Irwin et al. (1999), and Bowles et al. (2008) confirm the position of the band and its substructure; therefore, we eliminate this possibility.

2. The feature is real and is carried by $\mathrm{NH}_{3}$, but a wavelength calibration error in the WISE $1828+2650$ spectrum has shifted the feature blueward. We eliminate this as a possibility because other Y dwarf G102 spectra reduced by Schneider et al. (2015) in the same manner do not appear discrepant with the spectra of other $\mathrm{T} / \mathrm{Y}$ dwarfs.

3. The feature is not real and is an artifact of the data reduction. Such an "absorption feature" could arise in a low $\mathrm{S} / \mathrm{N}$ spectrum if a cluster of bad or noisy pixels conspires to produce an emission-like feature at $\sim 1.0 \mu \mathrm{m}$ thereby creating an apparent absorption feature at $\sim 1.03 \mu \mathrm{m}$. In this case, the identification of the absorption feature would be a result of our a priori expectations of finding $\mathrm{NH}_{3}$ absorption. Five individual spectra are combined to produce the final spectrum, and we can visually identify the absorption feature in four of the five spectra, so we eliminate this possibility.

4. The feature is real but its carrier is a molecule other than $\mathrm{NH}_{3}$. Since the wavelengths of the $\mathrm{NH}_{3}$ absorption coefficients are correct, and we cannot identify an error in our data reduction, we conclude that the feature is probably real but is carried by a molecule other than $\mathrm{NH}_{3}$. We explore this possibility in more detail presently.

In a search for the carrier of this feature, we identified an absorption feature in the reflected-light spectra of Jupiter, Saturn, Uranus, and Neptune (Rayner et al. 2009) whose rough central wavelength and width match that of the unidentified feature (see right panel of Figure 3). The presence of this band in all of the solar system giant planets strongly suggests the carrier is methane, as ammonia is sequestered well below the clouds in Uranus and Neptune. Indeed a comparison of the giant planet spectra to models generated with and without $\mathrm{CH}_{4}$ opacity also indicates that the feature is carried by $\mathrm{CH}_{4}(\mathrm{P}$. Irwin, private communication). The third and fourth panels of the left column of Figure 3 therefore show the cross-section spectrum of $\mathrm{CH}_{4}$ at $T=100$ and $300 \mathrm{~K}(P=0.1$ bar $)$ from Yurchenko \& Tennyson (2014) and Irwin et al. (2010); they have been smoothed to $R=210$ and resampled onto the wavelength grid of the WISE $1828+2650$ spectrum. The $T=100 \mathrm{~K}$ Yurchenko \& Tennyson (2014) spectrum provides the best match to the structure of the absorption feature. In particular, the double-peaked cross-section spectrum matches the structure of the absorption band, and the "emission-like" feature at $\sim 1.0 \mu \mathrm{m}$ is naturally explained by the lack of opacity at that wavelength. However, the Yurchenko \& Tennyson band is shifted slightly redward of our feature, which, taken at face value, weakens the case for the feature being carried by $\mathrm{CH}_{4}$. Interestingly, the position of the Irwin et al. band does not match that of the Yurchenko \& Tennyson band, which suggests some uncertainty in the theoretical position of this band. The apparent match between the low-temperature methane opacity structure and the observed feature is puzzling. For a cloudless atmosphere, models indicate that the flux in this spectral region emerges from quite deep in the atmosphere, where the local temperature is around $800 \mathrm{~K}$. If the carrier of the feature is methane, this may be an indication that water clouds, which form around $300 \mathrm{~K}$, are limiting the depth of the visible atmosphere here. A complete analysis of this will be the subject of future work.

\subsection{Spectral Classification}

WISE $1828+2650$ was originally classified as $>$ Y0 by Cushing et al. (2011) because its $1.1-1.7 \mu \mathrm{m}$ spectrum exhibited unique characteristics unseen in the spectra of latetype $\mathrm{T}$ dwarfs and $\mathrm{Y} 0$ dwarfs. Its type was later revised to $\geqslant \mathrm{Y} 2$ after the discovery of the Y1 dwarf WISE 035000.32-565830.2 (Kirkpatrick et al. 2012). With an improved spectrum in hand, we can return to the question of its spectral type.

Figure 4 shows a sequence of late-type $\mathrm{T}$ and $\mathrm{Y}$ dwarf spectra all observed with the G102+G141 WFC3 grism combination (Schneider et al. 2015; this work). The new WISE $1828+2650$ spectrum is still clearly distinct from that of the T dwarfs, and therefore the spectral type should remain a Y dwarf. Trends in spectral morphology at the T/Y boundary noted by Kirkpatrick et al. (2012) and Schneider et al. (2015) continue with the addition of the WISE $1828+2650$ spectrum: (1) the $Y$-band peak becomes more symmetrical, (2) the $Y$-band peak wavelength becomes bluer, (3) the $H$-band peak becomes more symmetrical, and (4) with the exception of WISEA J053516.87-750024.6 (whose G102 spectrum may still be contaminated; Schneider et al. 2015), the $Y$-, $J$-, and $H$-band peaks evolve toward having equal intensities. Interestingly, the 

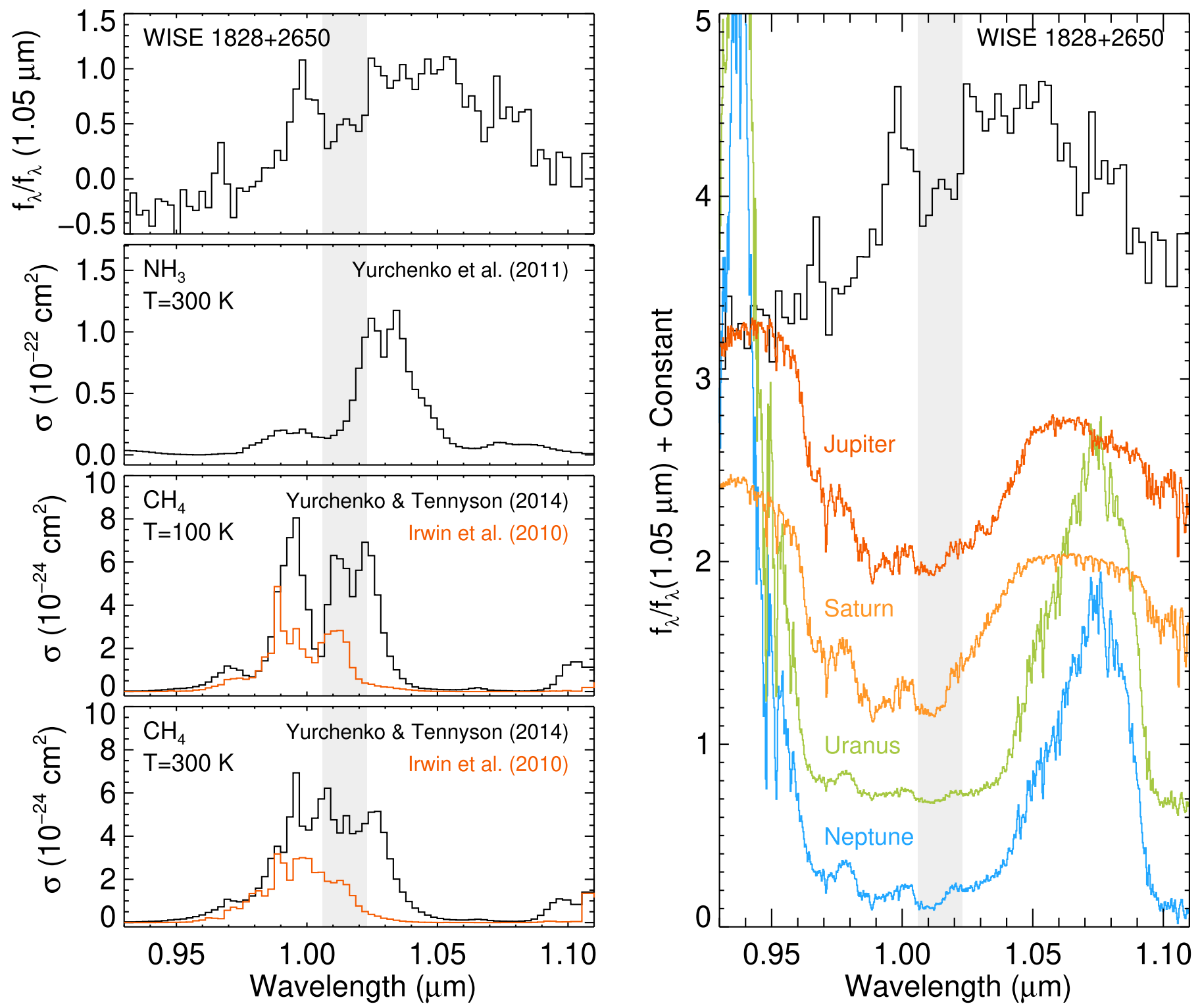

Figure 3. Left: $Y$-band spectrum of WISE $1828+2650$ with the unknown absorption feature highlighted in light gray along with the cross sections of $\mathrm{CH}_{4}$ and $\mathrm{NH}_{3}$ smoothed to the resolving power of $R=210$ and resampled onto the wavelength grid of the WISE $1828+2650$ spectrum. Right: $Y$-band spectrum of WISE 1828 +2650 with the unknown absorption feature highlighted in light gray along with the reflected-light spectra of the gas giant planets from Rayner et al. (2009). The giant planet spectra are at $R=2000$.

tentative new $\mathrm{CH}_{4}$ band appears weakly in the spectrum WISEA J053516.87-750024.6, although the $\mathrm{S} / \mathrm{N}$ is quite low. Since the spectrum of WISE $1828+2650$ smoothly extends the $\mathrm{Y}$ dwarf spectral sequence beyond Y1, its spectral type should remain $\geqslant Y 2$ until the near-infrared spectra of cooler objects like WISE 0855-0714 are obtained.

\subsection{The Spectral Energy Distribution}

Figure 5 shows the spectral energy distribution of WISE $1828+2650$ constructed by combining the new HST spectrum and the Maunakea Observatories (MKO) $K$-band (Leggett et al. 2013), Spitzer [3.6] and [4.5] (Kirkpatrick et al. 2019), and CatWISE2020 $W 1$ and $W 2$ (Marocco et al. 2021) photometric points. The apparent magnitudes were converted to average flux densities and plotted at the wavelengths of $\lambda_{\text {iso }}^{K}=$ $2.198 \mu \mathrm{m}, \quad \lambda_{0}^{[3.6]}=3.544 \mu \mathrm{m}, \quad \lambda_{0}^{[4.5]}=4.487 \mu \mathrm{m}, \quad$ and $\lambda_{\text {iso }}^{W 1}=$ $3.3526 \mu \mathrm{m}, \quad \lambda_{\text {iso }}^{W 2}=4.602 \mu \mathrm{m}$ using the zero points and wavelengths in Tokunaga \& Vacca (2005), Reach et al. (2005), and Jarrett et al. (2011), for the MKO, Spitzer, and WISE photometry, respectively. For clarity, we do not include photometric measurements at wavelengths that are sampled by the HST spectrum. The large amount of flux emerging at [4.5]/ $W 2$ is a result of the fact that this wavelength range is relatively free of stronger absorbers like $\mathrm{CH}_{4}, \mathrm{NH}_{3}$, and $\mathrm{H}_{2} \mathrm{O}$, which allows the observer to see deeper, and thus hotter, layers of the atmosphere where $T>T_{\text {eff. }}$.

\section{Physical Properties}

\subsection{Bolometric Luminosity}

A brown dwarf's bolometric luminosity is arguably the easiest fundamental parameter to measure since knowledge of only distance $d$ and bolometric flux $f_{\mathrm{bol}}$ is required. The spectral energy distributions of hotter $\mathrm{L}$ and early- to mid-type T dwarfs peak at red-optical and near-infrared wavelengths where 


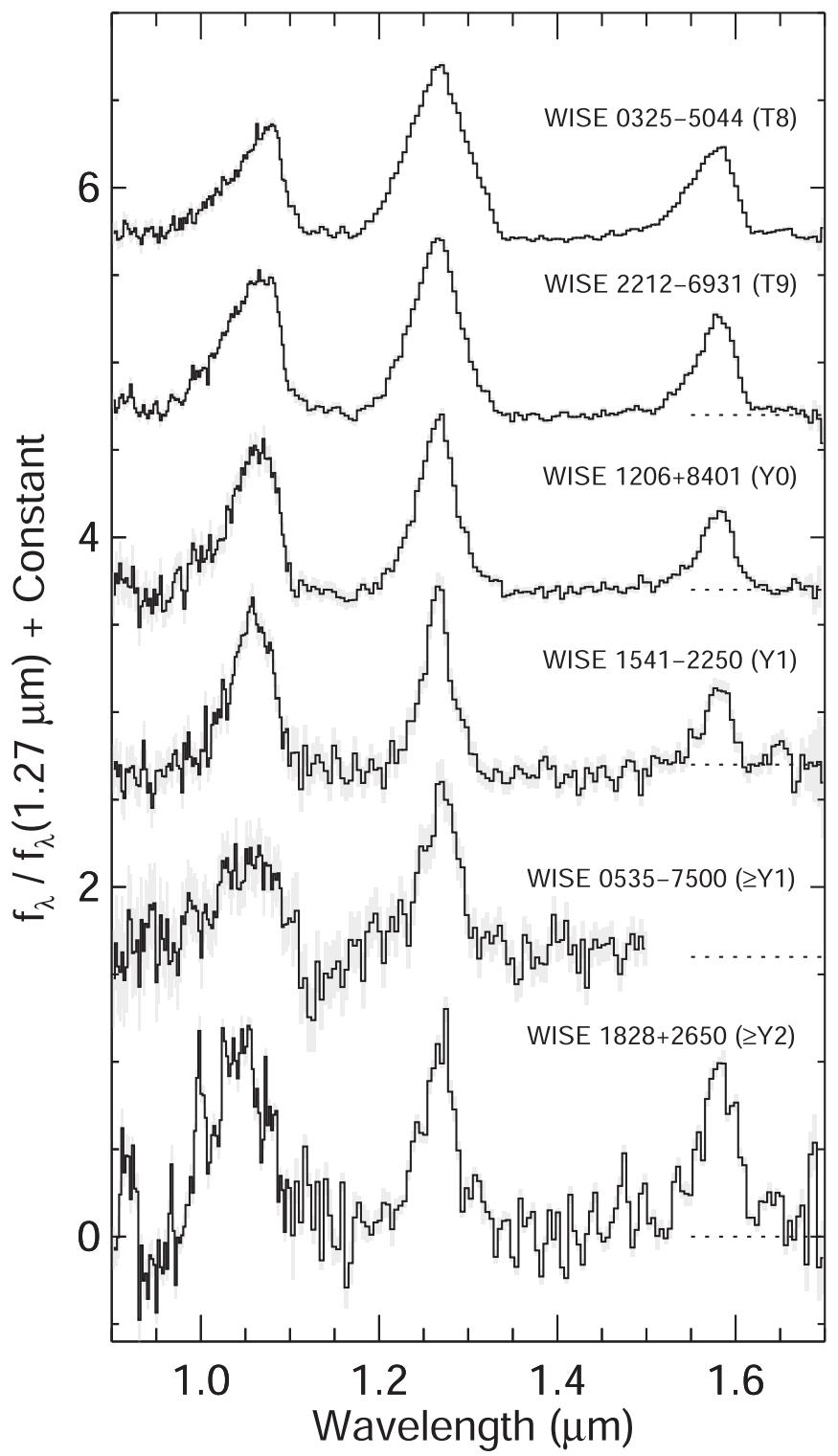

Figure 4. $0.9-1.7 \mu \mathrm{m}$ spectral sequence at the $\mathrm{T} / \mathrm{Y}$ boundary. The spectra of WISEA J032504.52-504403.0 (T8), WISEA J221216.27-693121.6 (T9), WISEA J120604.25+840110.5 (Y0), WISE J154151.65-225024.9 (Y1), and WISEA J053516.87-750024.6 ( $\geqslant$ Y1) are from Schneider et al. (2015) while the spectrum of WISE $1828+2650$ is from this work. The $H$-band spectrum of WISEA J053516.87-750024.6 is missing because it was contaminated by first-order light from a nearby star (Schneider et al. 2015). Uncertainties for each spectrum are shown as gray bars.

spectra are easily obtained from the ground. Integration of a spectral energy distribution consisting of a red-optical and nearinfrared spectrum, an $L$-band photometric point and/or WISE $W 1$ and $W 2$ points, and a Rayleigh-Jeans tail to account for (unobserved) emergent flux at longer wavelengths therefore provides a reasonably accurate estimate of $F_{\text {bol }}$ (Cushing et al. 2006; Filippazzo et al. 2015). However, the shift in the peak of the Planck function to longer wavelengths for the cooler latetype $\mathrm{T}$ dwarfs and $\mathrm{Y}$ dwarfs complicates the calculation of their bolometric fluxes because more and more energy emerges at wavelengths that are either difficult or impossible to access with current instrumentation (Marley \& Leggett 2009).

We constructed a continuous spectral energy distribution of WISE $1828+2650$ as follows. We first assumed that no flux emerges at wavelengths shortward of $0.90 \mu \mathrm{m}$ and then extended the HST spectrum by piecewise linear interpolation through the $K$-band, $W 1,[3.6],[4.5]$, and $W 2$ photometric points. WISE $1828+2650$ has $W 3=12.444 \pm 0.338(\mathrm{~S} / \mathrm{N}=$ 3.2; Cutri et al. 2013), but visual inspection of the atlas tile shows no clear source. We therefore do not use it or the W4 upper limit in the construction of the spectral energy distribution. To account for the flux at wavelengths longer than $W 2$, we extend a Rayleigh-Jeans tail from the flux density point at $4.602 \mu \mathrm{m}$ (the isophotal wavelength of the $W 2$ filter) to $\lambda=\infty$. Integrating over the spectral energy distribution, we measure a bolometric flux of $f_{\mathrm{bol}}=(1.015 \pm 0.01) \times 10^{-16} \mathrm{~W}$ $\mathrm{m}^{-2}$, which is the mean and standard deviation of 10,000 Monte Carlo realizations of $f_{\text {bol }}$, which account for the $2 \%$ uncertainty in the absolute flux calibration of the HST spectrum and the uncertainties in the individual spectral and photometric points. Using a relative parallax of $\pi=100.7 \pm 2.3$ mas (Kirkpatrick et al. 2019), we find $\log \left(L_{\text {bol }} / \mathcal{L}_{\odot}^{N}\right)=-6.50 \pm$ 0.02 , where $\mathcal{L}_{\odot}^{N}$ is the International Astronomical Union nominal solar luminosity of $3.828 \times 10^{26} \mathrm{~W}$ (Mamajek et al. 2015). We urge caution when using the $f_{\text {bol }}$ and $L_{\text {bol }} / \mathcal{L}_{\odot}^{N}$ measurements because they are likely dominated by systematic uncertainties due to the fact that the emergent spectra of cool brown dwarfs at wavelengths longward of $W 2$ are not Planck functions (e.g., Burrows et al. 2003; Cushing et al. 2006), and the Rayleigh-Jeans tail extension contributes $\sim 62 \%$ of the total bolometric flux in units of $f_{\lambda}$.

Dupuy \& Kraus (2013) computed the bolometric luminosity of WISE 1828+2650 using near-infrared and Spitzer Space Telescope/Infrared Array Camera (IRAC) photometry and model atmospheres to account for the emergent flux at other wavelengths. Interestingly, they measured a value of $\log \left(L_{\text {bol }} / L_{\odot}\right)=-6.13_{-0.16}^{+0.20}$, which is significantly brighter than our value, even when taking into account the uncertainties in our respective measurements. Our bolometric flux measurements agree within the uncertainties, and so the difference in bolometric luminosities can be almost completely explained by the difference in parallaxes used to compute the distance to WISE $1828+2650,70 \pm 14$ mas in the case of Dupuy \& Kraus and $100.7 \pm 2.3$ mas in the case of Kirkpatrick et al. The relative uncertainty in the Kirkpatrick et al. parallax is a factor of nine lower than the Dupuy \& Kraus parallax; so hereafter, we will use our value of $\log \left(L_{\text {bol }} / \mathcal{L}_{\odot}^{N}\right)$.

\subsection{Effective Temperature}

Estimating the effective temperature of WISE $1828+2650$ has proven difficult given its intrinsic faintness and contaminated spectrum. Cushing et al. (2011) estimated the effective temperature to be less than $300 \mathrm{~K}$ based on the near equal height of the spectral peaks in the $J$ and $H$ bands and its extreme $J-W 2$ color while Leggett et al. (2013) suggest that WISE $1828+2650$ is actually a $\sim 2$ Gyr old binary with $M=$ $10,7 M_{\text {Jup }}, T_{\text {eff }}=325,300 \mathrm{~K}$, and $\log g=4.5,4.0\left[\mathrm{~cm} \mathrm{~s}^{-2}\right]$. Beichman et al. (2013) fit multiband absolute photometry in various combinations and found $T_{\text {eff }} \sim 250-450 \mathrm{~K}$ but noted that the "estimated physical parameters should be taken with a grain of salt" given how poorly the models fit the data. Dupuy \& Kraus (2013) used their bolometric luminosity measurement (see Section 4.1) and evolutionary models to estimate an effective temperature of $470-640 \mathrm{~K}$ for ages between 1 and $5 \mathrm{Gyr}$, but this rather high value is a result of their relatively low-precision parallax measurement. 


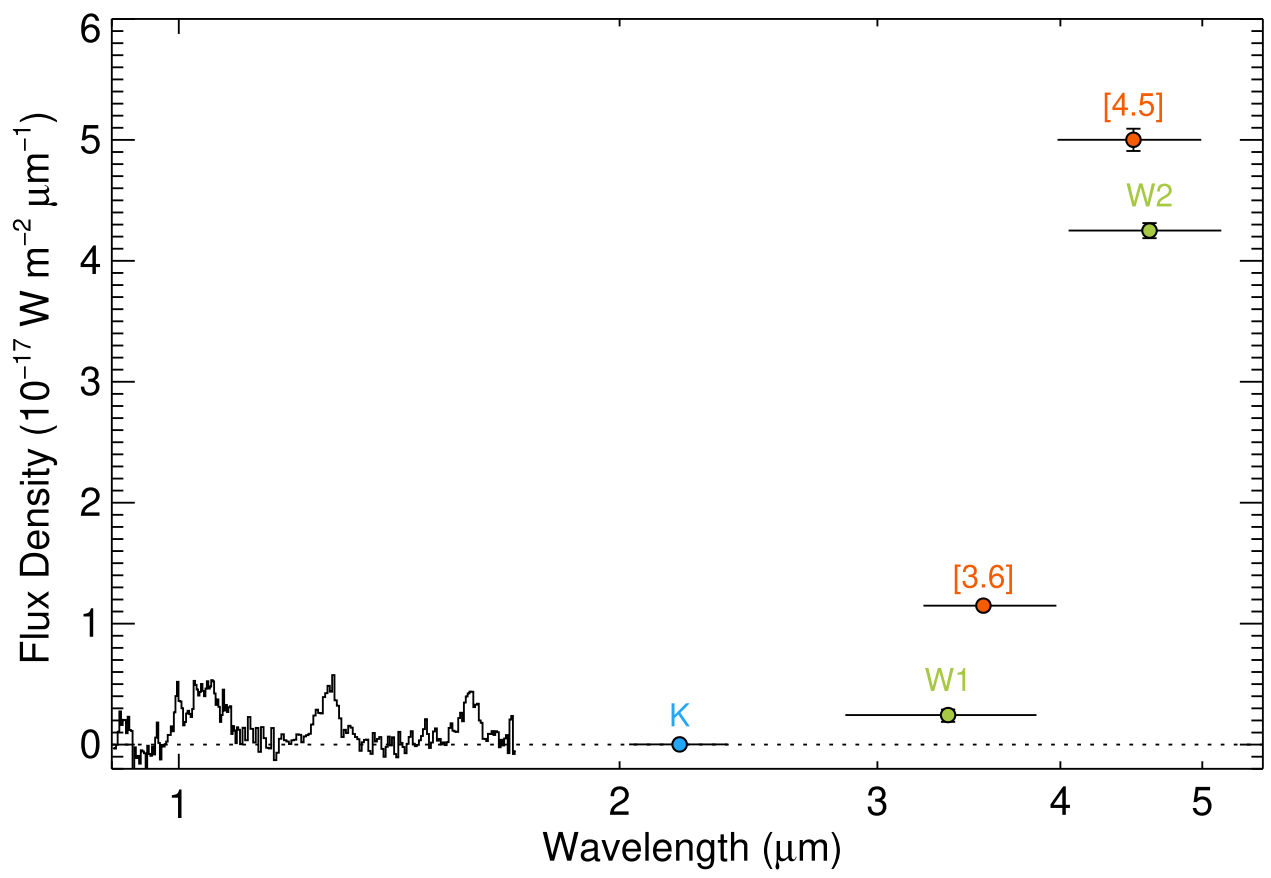

Figure 5. Spectral energy distribution of WISE 1828+2650. The spectrum (black) is from this work, while the $K$-band photometric point is from Leggett et al. (2013), the CatWISE2020 W1 and W2 photometry is from Marocco et al. (2021), and the Spitzer [3.6] and [4.5] photometry is from Kirkpatrick et al. (2019). The photometry is plotted at the nominal or isophotal filter wavelengths, and the widths of the filters are denoted with horizontal lines.

We can make a zeroth-order estimate of the effective temperature of WISE $1828+2650$ by making the reasonable assumption that its radius is $R \approx 1 \mathcal{R}_{\mathrm{J}}^{\mathrm{N}}$-the nominal value for Jupiter's equatorial radius of $7.1492 \times 10^{7} \mathrm{~m}$ (Mamajek et al. 2015) - since the radii of all evolved brown dwarfs are with $\sim 30 \%$ of this value due to the competing effects of Coulomb and electron degeneracy effects (Burrows et al. 2001). With $T_{\text {eff }}=\left(d^{2} f_{\text {bol }} / \sigma R^{2}\right)^{-1 / 4}$, where $\sigma$ is the Stefan-Boltzmann constant, we find $T_{\text {eff }}=426 \mathrm{~K}$. If WISE $1828+2650$ is an equal-luminosity binary, the effective temperature of each component would be $T_{\text {eff }}=351 \mathrm{~K}$. Both values are consistent with previous estimates (with the exception of the Dupuy $\&$ Kraus estimate, which is the result of their large bolometric luminosity measurement) confirming that WISE $1828+2650$ is still one of the coolest brown dwarfs known, especially if it is a binary. In the following two sections, we make additional estimates of the effective temperature of WISE $1828+2650$, using both evolutionary and atmospheric models.

\subsubsection{Bolometric Luminosity and Evolutionary Models}

With an estimate of WISE 1828+2650's bolometric luminosity, we can use evolutionary models to estimate its effective temperature following the method described by Saumon et al. (2000). Figure 6 shows the evolution of solar metallicity cloudless brown dwarfs in the effective temperature/surface gravity plane (Marley et al. 2020). The locus of points with bolometric luminosities equal to that of WISE 1828 +2650 for ages between 0.1 and $10 \mathrm{Gyr}$ is shown as a nearvertical blue line, which constrains its effective temperature to lie between $386 \leqslant T_{\text {eff }} \leqslant 461 \mathrm{~K}$. This is significantly lower than the value found by Dupuy \& Kraus (2013) using a similar technique, but as noted previously, this is simply a result of using a different parallax value to compute the bolometric luminosity.

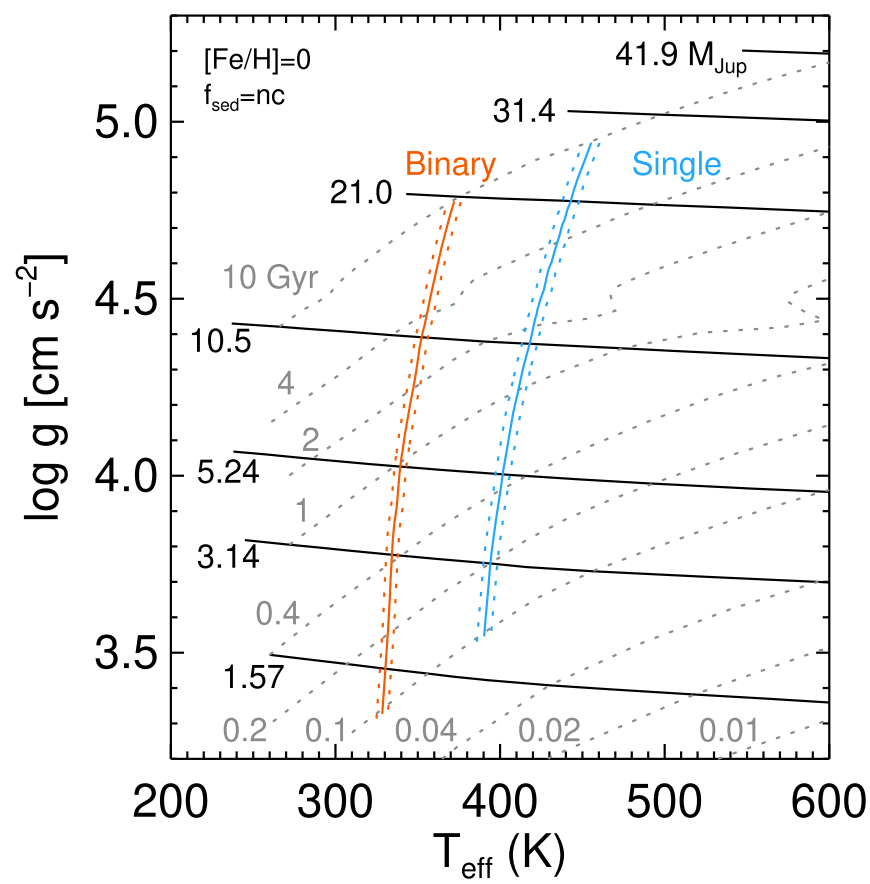

Figure 6. Evolution of Bobcat Sonora solar metallicity cloudless brown dwarfs in the effective temperature surface gravity plane (Marley \& Saumon 2020; Marley et al. 2021). The black lines are cooling tracks for brown dwarfs with masses of $41.9,31.4,21,10.5,5.24,3.14$, and $1.57 \mathcal{M}_{\mathrm{J}}^{N}$, while the gray lines are isochrones for ages of $10,4,2,1,0.4,0.2,0.1,0.04,0.02$, and $0.01 \mathrm{Gyr}$. The loci of points with bolometric luminosities equal to that of WISE 1828 +2650 for ages between 0.1 and $10 \mathrm{Gyr}$ are shown as the solid near-vertical lines while the $\pm 1 \sigma$ uncertainties on the bolometric luminosities are shown as dotted lines.

The near-vertical red line corresponds to the locus of points with bolometric luminosities equal to half that of WISE 1828 +2650 , which is appropriate if it is an equal-brightness binary. In this case, the effective temperature of the two components 
must lie between $325 \leqslant T_{\text {eff }} \leqslant 376 \mathrm{~K}$, which is more consistent with previous estimates based on analyses of the colors and spectral energy distribution of WISE $1828+2650$.

\subsubsection{Atmospheric Models}

We also estimate the effective temperature of WISE 1828 +2650 by fitting the HST spectrum and spectral energy distribution shown in Figure 5 with two grids of atmospheric models: (1) the Morley et al. (2014) models that include the formation of water clouds and (2) the cloudless Marley et al. (2018) Sonora Bobcat models. The solar metallicity, partly cloudy $\left(f_{\text {sed }}=5, h=0.5\right)^{11}$ Morley et al. grid is a function of two free parameters, $\boldsymbol{\theta}_{\text {atm }}=\left\{T_{\text {eff }}, g\right\}$, with $T_{\text {eff }}=200-450 \mathrm{~K}$ in steps of $25 \mathrm{~K}$ (except for $T_{\text {eff }}=425 \mathrm{~K}$ ), $\log g=3.5,4.0,4.5$, $5.0\left(\mathrm{~cm} \mathrm{~s}^{-2}\right)$, for a total of 40 models. The cloudless $\left(f_{\mathrm{sed}}=\mathrm{nc}\right)$ Sonora Bobcat grid is a function of four free parameters, $\boldsymbol{\theta}_{\mathrm{atm}}=\left\{T_{\mathrm{eff}}, g,[\mathrm{M} / \mathrm{H}],[\mathrm{C} / \mathrm{O}]\right\}$, with $T_{\text {eff }}=250-450 \mathrm{~K}$ in steps of $50 \mathrm{~K}, \log g=4.0,4.5\left[\mathrm{~cm} \mathrm{~s}^{-2}\right],[\mathrm{M} / \mathrm{H}]=+0.5,0.0,-0.5$, and $[\mathrm{C} / \mathrm{O}]=0.0,-0.3,-0.6^{12}$ for a total of 72 models. We note that the $[\mathrm{M} / \mathrm{H}]$ and $[\mathrm{C} / \mathrm{O}]$ values are for the bulk composition of the atmosphere and do not necessarily reflect the composition of the gas phase because condensate species such as enstatite $\left(\mathrm{MgSiO}_{3}\right)$ and forsterite $\left(\mathrm{Mg}_{2} \mathrm{SiO}_{4}\right)$ form at these temperatures and pressures.

One-dimensional model atmospheres provide flux densities at the surface of the brown dwarf at $n$ discrete wavelengths, i.e., $\mathcal{F}_{\lambda}\left(\boldsymbol{\theta}_{\text {atm }}\right)=\left[\mathcal{F}_{\lambda}\left(\boldsymbol{\theta}_{\text {atm }}, \lambda_{1}\right), \mathcal{F}_{\lambda}\left(\boldsymbol{\theta}_{\text {atm }}, \lambda_{2}\right), \mathcal{F}_{\lambda}\left(\boldsymbol{\theta}_{\text {atm }}, \lambda_{3}\right), \ldots\right.$, $\left.\mathcal{F}_{\lambda}\left(\theta_{\text {atm }}, \lambda_{n}\right)\right]$. In order to compare each of the $j$ models in a given grid to the observations of WISE $1828+2650$, we must simulate observing the model spectra with the techniques used to collect our data. The spectroscopic portion of the data is given by,

$$
\mathcal{M}_{\lambda}^{\mathrm{HST}}\left(\boldsymbol{\theta}_{\mathrm{atm}, j}\right)=h(\lambda) * K(\lambda) * \mathcal{F}_{\lambda}\left(\boldsymbol{\theta}_{\mathrm{atm}, j}\right),
$$

where $K(\lambda)$ is a Gaussian kernel that smooths the $j$ th model $\mathcal{F}_{\lambda}\left(\theta_{\mathrm{atm}, j}\right)$ to the resolving power of the data, $h(\lambda)$ is a resampling kernel that resamples the smoothed model spectrum onto the wavelength grid of the data, and ' $*$ ' denotes a convolution. The resolving powers of the HST spectrum are given in Section 2, and we used a linear interpolation kernel for $h(\lambda)$.

The photometric portions of the data can be simulated by integrating the model spectra over the appropriate bandpasses. The MKO $K$-band point is given by,

$$
\mathcal{M}_{\lambda}^{K}\left(\boldsymbol{\theta}_{\mathrm{atm}, j}\right)=\frac{\int \lambda \mathcal{F}_{\lambda}\left(\boldsymbol{\theta}_{\mathrm{atm}, j}, \lambda\right) S(\lambda) d \lambda}{\int \lambda S(\lambda) d \lambda},
$$

where $S(\lambda)$ is the system response function of the $K$ band, which we assume to be given by the product of the filter transmission and the typical atmospheric transmission at an air mass of 1 . The $\lambda$ inside the integral converts the energy flux densities to photon flux densities, which ensures that the integrated fluxes are proportional to the observed photon count

\footnotetext{
${ }^{11} f_{\text {sed }}$ is the sedimentation parameter, which describes the efficiency of condensate sedimentation (Ackerman \& Marley 2001), and $h$ is a parameter that ranges from 0 to 1 that describes the fraction of the atmosphere that can be described by a cloud-free column (Marley et al. 2010). Larger values of $f_{\text {sed }}$ imply thinner clouds, and a value of $h=0.5$ implies a $50 \%$ cloud coverage.

12 The model file naming convention is that the solar $\mathrm{C} / \mathrm{O}$ ratio is denoted as $\mathrm{C} / \mathrm{O}=1$, which we write here as $[\mathrm{C} / \mathrm{O}]=0.0$.
}

rate. The [3.6] and [4.5] IRAC points are given by,

$$
\mathcal{M}_{\nu}^{\mathrm{IRAC}}\left(\boldsymbol{\theta}_{\mathrm{atm}, j}\right)=\frac{\int\left(\nu_{0} / \nu\right) \mathcal{F}_{\nu}\left(\boldsymbol{\theta}_{\mathrm{atm}, j}, \nu\right) S(\nu) d \nu}{\int\left(\nu_{0} / \nu\right)^{2} S(\nu) d \nu},
$$

where $\nu_{0}$ is the nominal frequency, and where $S(\nu)$ is the system response function of the telescope plus instrument plus detector system in units of $e^{-}$per photon (Cushing et al. 2006). The per-frequency flux density is then converted to a perwavelength flux density $\mathcal{M}_{\lambda}^{\mathrm{IRAC}}\left(\boldsymbol{\theta}_{\mathrm{atm}, j}\right)$ at the nominal wavelengths of the bandpasses. Finally, the WISE $W 1$ and $W 2$ points are given by,

$$
\mathcal{M}_{\lambda}^{\mathrm{WISE}}\left(\boldsymbol{\theta}_{\mathrm{atm}, j}\right)=\frac{\int \mathcal{F}_{\lambda}\left(\boldsymbol{\theta}_{\mathrm{atm}, j}, \lambda\right) S(\lambda) d \lambda}{\int S(\lambda) d \lambda}
$$

where $S(\lambda)$ is the system response functions. Note that we do not include the factor of $\lambda$ found in the integral of Equation (2), because the filter transmission curves have already been multiplied by $\lambda$. The $j$ th model at the surface of the brown dwarfs is then given by,

$$
\begin{gathered}
\mathcal{M}\left(\boldsymbol{\theta}_{\mathrm{atm}, j}\right)=\left[\mathcal{M}_{\lambda}^{\mathrm{HST}}\left(\boldsymbol{\theta}_{\mathrm{atm}, j}\right), \mathcal{M}_{\lambda}^{K}\left(\boldsymbol{\theta}_{\mathrm{atm}, j}\right),\right. \\
\left.\mathcal{M}_{\lambda}^{[3.6]}\left(\boldsymbol{\theta}_{\mathrm{atm}, j}\right), \mathcal{M}_{\lambda}^{[4.5]}\left(\boldsymbol{\theta}_{\mathrm{atm}, j}\right), \mathcal{M}_{\lambda}^{W 2}\left(\boldsymbol{\theta}_{\mathrm{atm}, j}\right)\right] .
\end{gathered}
$$

In order to compare a model spectral energy distribution $\mathcal{M}\left(\theta_{\mathrm{atm}, j}\right)$ to the data, we first need to multiple it by $(R / d)^{2}$, where $R$ is the radius of the brown dwarf and $d$ is its distance, in order to convert the flux densities at the surface of the brown dwarf to flux densities observed on or near Earth. While the distance to WISE $1828+2650$ is known to be $9.93 \mathrm{pc}$ (Kirkpatrick et al. 2019), its radius is unknown, and so we can only compare the relative shapes of the model spectra and data. We identify the best-fitting model spectrum in each grid following Cushing et al. (2008) where for each model $j$, we compute a $\chi^{2}$ statistic,

$$
\chi_{j}^{2}=\sum_{i=1}^{n}\left[\frac{f_{i}-C_{j} \mathcal{M}_{i}\left(\boldsymbol{\theta}_{\mathrm{atm}, j}\right)}{s_{i}}\right]^{2}
$$

where $n$ is the number of data points, $f_{i}$ and $\mathcal{M}_{j, i}$ are the flux densities of the data and $j$ th model, respectively, $s_{i}$ is the uncertainty in the $i$ th data point, ${ }^{13}$ and $C_{j}$ is an unknown multiplicative constant. For each model $j$, we determine the value of $C_{j}$ by minimizing $\chi^{2}$ with respect to $C_{j}$ to give,

$$
C_{j}=\frac{\sum_{i} f_{i} \mathcal{M}_{j, i} / s_{i}^{2}}{\sum_{i} \mathcal{M}_{j, i} / s_{i}^{2}}
$$

The best-fitting model in each grid is that with the lowest $\chi^{2}$ value. We fit both grids of model spectra to the HST spectrum and then to the entire spectral energy distribution (the HST spectrum and photometry), and the results are given in Columns $1-8$ in Table 3 and shown in Figure 7.

The atmospheric parameters of the best-fit model spectrum can be used to infer additional properties of WISE $1828+2650$ when they are combined with evolutionary models because the models provide a unique mapping between the structural

\footnotetext{
13 The standard deviation in the $\chi^{2}$ statistic requires knowledge of the uncertainty in our model, which is beyond the scope of this work. We therefore assume that the model standard deviations are given by the uncertainties on our data points.
} 
Table 3

WISE $1828+2650$ Model Fits

\begin{tabular}{|c|c|c|c|c|c|c|c|c|c|c|c|c|}
\hline \multirow[b]{2}{*}{$\begin{array}{l}\text { Models } \\
\text { (1) }\end{array}$} & \multicolumn{6}{|c|}{ Best-fit Atmospheric Parameters ${ }^{\mathrm{a}}$} & \multicolumn{4}{|c|}{ Evolution Parameters ${ }^{\mathrm{b}}$} & \multicolumn{2}{|c|}{ Estimated $^{\mathrm{c}}$} \\
\hline & $\begin{array}{l}T_{\text {eff }} \\
(\mathrm{K}) \\
(2)\end{array}$ & $\begin{array}{c}\log g \\
\left(\mathrm{~cm} \mathrm{~s}^{-2}\right) \\
(3)\end{array}$ & $\begin{array}{c}{[\mathrm{Fe} / \mathrm{H}]} \\
(5)\end{array}$ & $\begin{array}{c}\mathrm{C} / \mathrm{O}] \\
(6)\end{array}$ & $\begin{array}{l}C \\
\text { (7) }\end{array}$ & $\begin{array}{c}\chi^{2} / \text { dof } \\
(8)\end{array}$ & $\begin{array}{c}M \\
\left(\mathcal{M}_{\mathrm{J}}^{\mathrm{N}}\right) \\
(9)\end{array}$ & $\begin{array}{c}R \\
\left(\mathcal{R}_{\mathrm{J}}^{\mathrm{N}}\right) \\
(10)\end{array}$ & $\begin{array}{c}\tau \\
(\mathrm{Gyr}) \\
(11)\end{array}$ & $\begin{array}{c}\log L / L \odot \\
(12)\end{array}$ & $\begin{array}{c}d_{C} \\
(\mathrm{pc}) \\
(13)\end{array}$ & $\begin{array}{c}R_{C} \\
\left(\mathcal{R}_{\mathrm{J}}^{\mathrm{N}}\right) \\
(14)\end{array}$ \\
\hline \multicolumn{13}{|c|}{ HST Spectrum } \\
\hline Morley et al. (2014) & 350 & 5.0 & $0.0^{\mathrm{d}}$ & $0.0^{\mathrm{d}}$ & $2.7070 \times 10^{-20}$ & $5058 / 211$ & 29.6 & 0.85 & $26.7^{\mathrm{e}}$ & -6.98 & 12.0 & 0.71 \\
\hline Sonora Bobcat & 400 & 4.5 & -0.5 & 0.0 & $1.6848 \times 10^{-20}$ & $4474 / 211$ & 13.0 & 1.00 & 3.14 & -6.61 & 17.9 & 0.56 \\
\hline \multicolumn{13}{|c|}{ Spectral Energy Distribution } \\
\hline Morley et al. (2014) & 275 & 4.5 & $0.0^{\mathrm{d}}$ & $0.0^{\mathrm{d}}$ & $4.8680 \times 10^{-19}$ & $9421 / 216$ & 12.3 & 0.98 & 12.3 & -7.29 & 3.24 & 2.99 \\
\hline Sonora Bobcat & 350 & 4.0 & -0.5 & -0.6 & $9.8445 \times 10^{-20}$ & $7839 / 216$ & 4.98 & 1.10 & 0.760 & -6.76 & 8.16 & 1.34 \\
\hline \multicolumn{13}{|c|}{ HST Spectrum (Binary Fit) ${ }^{\mathrm{e}}$} \\
\hline Morley et al. (2014) & 350,350 & $5.0,5.0$ & $0.0,0.0^{\mathrm{d}}$ & $0.0,0.0^{\mathrm{d}}$ & $3.6496 \times 10^{-36}$ & $5058 / 211$ & $29.6,29.6$ & $0.85,0.85$ & $26.7,26.7^{\mathrm{e}}$ & $-6.98,-6.98$ & 17.0 & $\cdots$ \\
\hline Sonora Bobcat & 400,400 & $4.5,4.5$ & $-0.5,-0.5$ & $0.0,0.0$ & $1.6360 \times 10^{-36}$ & $4474 / 211$ & $13.0,13.0$ & $1.00,1.00$ & $3.14,3.14$ & $-6.61,-6.61$ & 25.3 & $\cdots$ \\
\hline \multicolumn{13}{|c|}{ Spectral Energy Distribution (Binary Fit $)^{\mathrm{e}}$} \\
\hline Morley et al. (2014) & 275,275 & $4.5,4.5$ & $0.0,0.0^{\mathrm{d}}$ & $0.0,0.0^{\mathrm{d}}$ & $4.9947 \times 10^{-35}$ & $9421 / 216$ & $12.3,12.3$ & $0.98,0.98$ & $12.3,12.3$ & $-7.29,-7.29$ & 4.59 & $\cdots$ \\
\hline Sonora Bobcat & 300,350 & $4.0,4.0$ & $0.0,-0.5$ & $-0.3,-0.6$ & $1.2819 \times 10^{-35}$ & $7286 / 216$ & $4.65,4.75$ & $1.09,1.10$ & $1.41,0.760$ & $-7.04,-6.76$ & 9.03 & $\cdots$ \\
\hline
\end{tabular}

\section{Notes.}

${ }^{\text {a }}$ The Morley et al. models have $f_{\text {sed }}=5, h=0.5$, while the Sonora Bobcat models have $f_{\text {sed }}=$ nc.

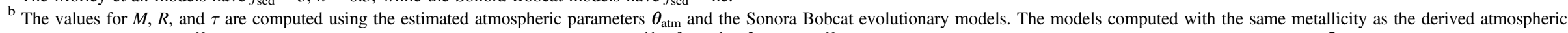
parameters were used. $\mathcal{M}_{\mathrm{J}}^{\mathrm{N}}$ is the nominal Jupiter mass (assuming $G=6.67430 \times 10^{-11} \mathrm{~m}^{3} \mathrm{~kg}^{-1} \mathrm{~s}^{-2}$ ) while $\mathcal{R}_{\mathrm{J}}^{\mathrm{N}}$ is the nominal value for Jupiter's equatorial radius of $7.1492 \times 10^{7} \mathrm{~m}$ (Mamajek et al. 2015).

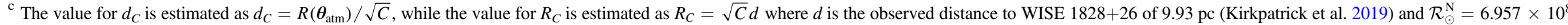
m. (Mamajek et al. 2015).

${ }^{\mathrm{d}}$ Not a free parameter in the fit.

e This value is an extrapolation beyond the $20 \mathrm{Gyr}$ limit of the evolution calculations. 

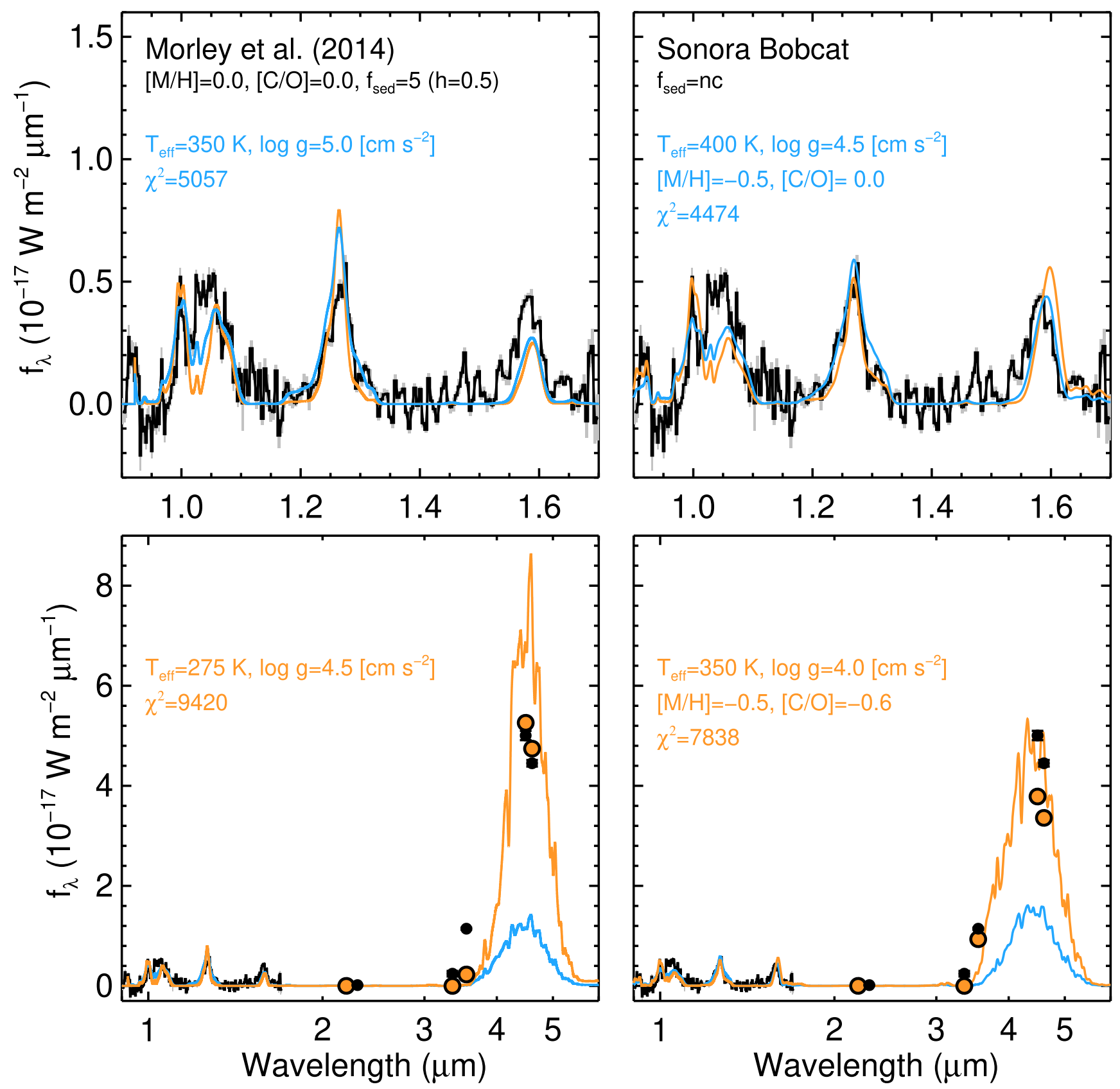

Figure 7. Best-fitting Morley et al. (2014) model spectra (left column) and Sonora models (right column). The top row shows the fits to the HST spectrum, while the bottom row shows the fits to the spectral energy distribution. The flux densities of WISE $1828+2650$ in the $K$ band, $W 1$, [3.6], [4.5], and $W 2$ are shown as filled black circles, while the flux densities of the models integrated over the corresponding bandpasses are shown as filled orange circles. The $K$-band model flux density has been slightly offset in wavelength for clarity. For comparison, the best-fitting model spectra to the HST spectrum are shown in the bottom panels, and the best-fitting model spectra to the spectral energy distribution are shown in the top panels.

parameters of radius $(R)$, mass $(M)$, age $(\tau)$, and luminosity $\left(L_{\mathrm{bol}}\right)$ and the atmospheric parameters of effective temperature and surface gravity; thus, Columns $9-11$ in Table 3 give the mass, radius, age, and luminosity of WISE $1828+2650$ according to the Bobcat Sonora evolutionary models (Marley $\&$ Saumon 2020; Marley et al. 2021). The scale factor $C$ is equal to $(R / d)^{2}$, and so with the evolutionary model radius $R\left(\boldsymbol{\theta}_{\mathrm{atm}}\right)$, we can empirically estimate the distance to WISE $1828+2650$ and compare it to the known distance of $d=9.93 \pm 0.23 \mathrm{pc}$ (Kirkpatrick et al. 2019). Alternatively, we can use the known distance to estimate its radius, which can then be compared to the predictions of evolutionary models. These estimates are given in Columns 13 and 14 of Table 3.
The location of the best-fit values in the effective temperature/ surface gravity plane with respect to the evolution of cloudless brown dwarfs is also shown in Figure 9.

Given that WISE $1828+2650$ is overluminous in nearly all color-magnitude diagrams, we also fit the data to model binary stars. For each grid, we simulated a collection of binary systems by scaling each of the model spectra by $R\left(\boldsymbol{\theta}_{\text {atm }}\right)^{2}$ (the radius given by the evolutionary models that corresponds to $\left.\boldsymbol{\theta}_{\text {atm }}\right)$ and then summed pairs of models $(k, l)$ as:

$$
\begin{gathered}
\mathcal{M}_{j}=R\left(\boldsymbol{\theta}_{\mathrm{atm}, k}\right)^{2}\left[\mathcal{M}_{\lambda}^{\mathrm{HST}}\left(\boldsymbol{\theta}_{\mathrm{atm}, k}\right), \mathcal{M}_{\lambda}^{K}\left(\boldsymbol{\theta}_{\mathrm{atm}, k}\right),\right. \\
\left.\mathcal{M}_{\lambda}^{[3.6]}\left(\boldsymbol{\theta}_{\mathrm{atm}, k}\right), \mathcal{M}_{\lambda}^{[4.5]}\left(\boldsymbol{\theta}_{\mathrm{atm}, k}\right), \mathcal{M}_{\lambda}^{W 2}\left(\boldsymbol{\theta}_{\mathrm{atm}, k}\right)\right]+
\end{gathered}
$$



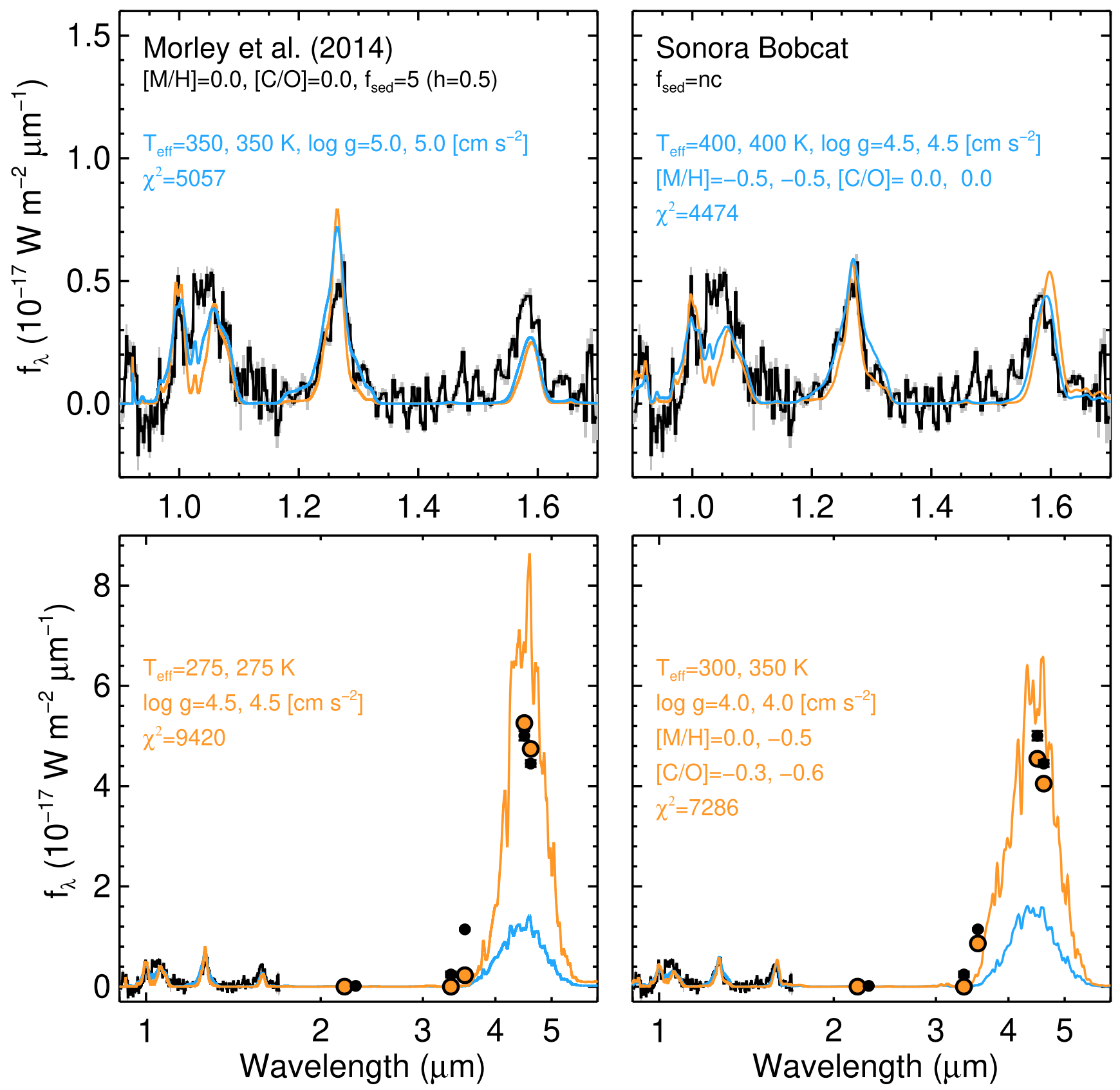

Figure 8. Similar to Figure 7, for the best-fitting Morley et al. (2014) binary model spectra (left column) and Sonora Bobcat binary model spectra (right column).

$$
\begin{aligned}
& R\left(\boldsymbol{\theta}_{\mathrm{atm}, l}\right)^{2}\left[\mathcal{M}_{\lambda}^{\mathrm{HST}}\left(\boldsymbol{\theta}_{\mathrm{atm}, l}\right), \mathcal{M}_{\lambda}^{K}\left(\boldsymbol{\theta}_{\mathrm{atm}, l}\right),\right. \\
& \left.\quad \mathcal{M}_{\lambda}^{[3.6]}\left(\boldsymbol{\theta}_{\mathrm{atm}, l}\right), \mathcal{M}_{\lambda}^{[4.5]}\left(\boldsymbol{\theta}_{\mathrm{atm}, l}\right), \mathcal{M}_{\lambda}^{W 2}\left(\boldsymbol{\theta}_{\mathrm{atm}, l}\right)\right] .
\end{aligned}
$$

However, we placed the following constraints,

$$
\begin{gathered}
\left|\tau_{k}-\tau_{l}\right| \leqslant 1 \mathrm{Gyr}, \\
\left|[\mathrm{Fe} / \mathrm{H}]_{k}-[\mathrm{Fe} / \mathrm{H}]_{l}\right| \leqslant 0.5 \mathrm{dex}, \\
\left|[\mathrm{C} / \mathrm{O}]_{k}-[\mathrm{C} / \mathrm{O}]_{l}\right| \leqslant 0.3 \mathrm{dex},
\end{gathered}
$$

on the components of the binary to ensure that they have similar compositions and ages. While it is reasonable to assume that the components of a binary have identical ages and compositions, the course sampling of the atmospheric parameters requires weakening this constraint in order to avoid creating only equal-parameter binaries. The results of these fits are given in Columns $1-8$ in Table 3 and shown in Figures 8 and 9. The corresponding structural parameters are given in Columns 9-12. Since the model spectra are first scaled by $R\left(\boldsymbol{\theta}_{\mathrm{atm}}\right)^{2}$, the scale factor is simply given by $C=(1 / d)^{2}$, and so we can provide an empirical estimate of the distance as $d_{C}=1 / \sqrt{C}$, and the results are given in Column 13 of Table 3 .

\section{Discussion}

Statistically speaking, all of the model spectra are poor fits to the data since the $\chi^{2}$ values of the fits are factors of 20-40 times larger than the number of degrees of freedom. Given the complexity of the model atmospheres, statistically poor fits to brown dwarf spectra are not uncommon, especially when the observations span a broad wavelength range (e.g., Cushing et al. 2008; 

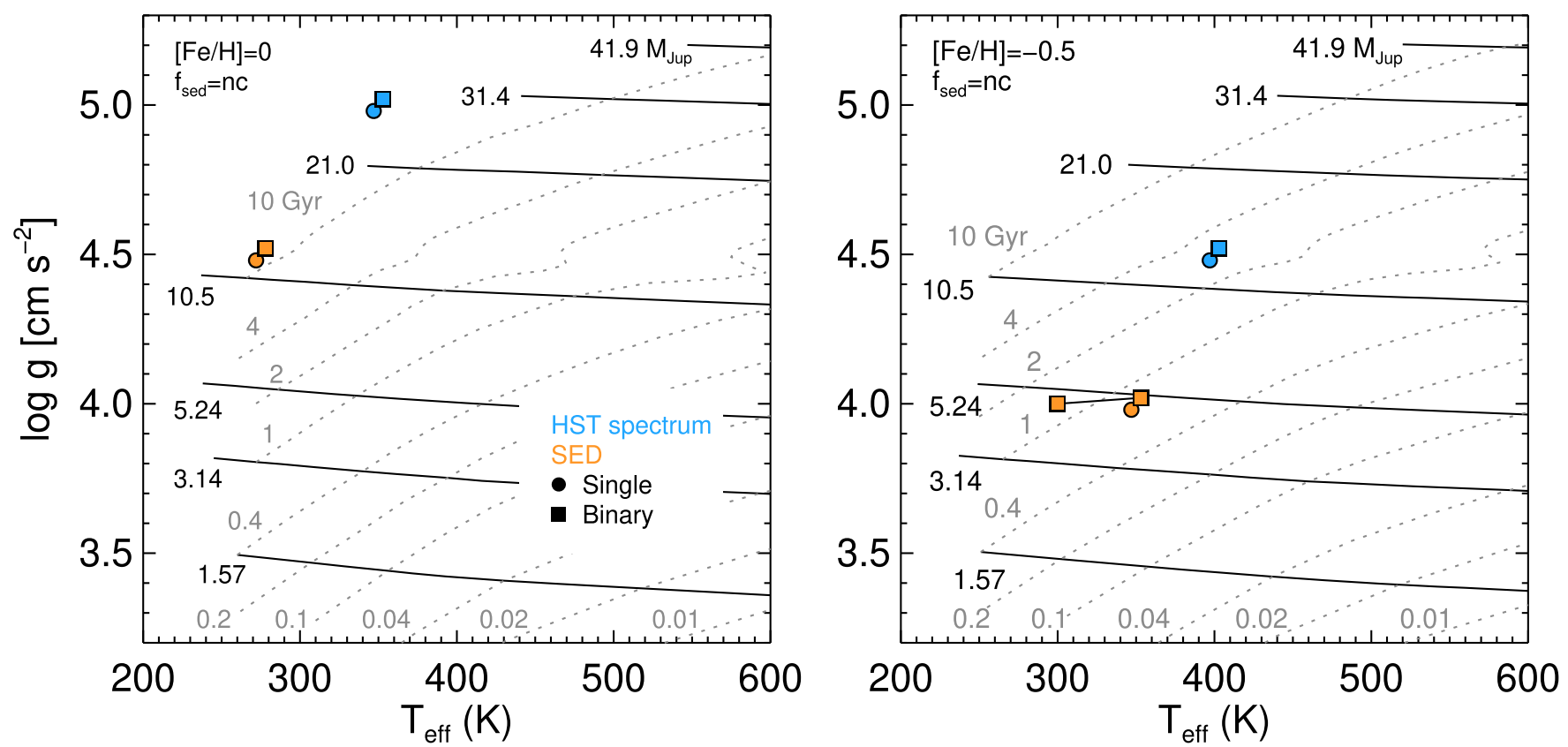

Figure 9. Evolution of solar metallicity (left) and $[\mathrm{Fe} / \mathrm{H}]=-0.5$ (right) cloudless brown dwarfs in the effective temperature surface gravity plane (Marley \& Saumon 2020; Marley et al. 2021). The black lines are cooling tracks for brown dwarfs with masses of 41.9, 31.4, 21, 10.5, 5.24, 3.14, and 1.57 $\mathcal{M}_{\mathrm{J}}^{N}$ while the gray lines are isochrones for ages of 10, 4, 2, 1, 0.4, 0.2, 0.1, 0.04, 0.02, and $0.01 \mathrm{Gyr}$. Also plotted are the results of the atmospheric model fits discussed in Section 4.2.2. The results of fits assuming WISE $1828+2650$ is a single object are shown as circles, while the results assuming it is binary are shown as squares. The results of fits to just the HST spectrum are shown in blue while the results for the spectral energy distribution are shown in orange. Finally, the points have been shifted slightly from their true values for visual clarity.

Stephens et al. 2009; Schneider et al. 2015; Luhman \& Esplin 2016; Morley et al. 2018). As a result, we do not attempt to provide statistical uncertainties on the derived parameters. Nevertheless, it is still instructive to take the fits at face value because they not only provide some insight into the physical properties of the brown dwarfs but they also allow us to identify wavelengths where the models need improvement.

The HST spectrum is best fit by the Sonora model spectrum (upper right panel of Figure 7) because it more accurately reproduces the heights of the $J$ - and $H$-band peaks. Both models fit the $Y$-band peak poorly because the $\mathrm{NH}_{3}$ band in both models is too strong. This is most likely a result of the fact that the atmospheric models assume equilibrium chemistry. It has been shown that vertical mixing within an atmosphere keeps the nitrogen chemistry from coming into equilibrium such that the abundance of $\mathrm{N}_{2}$ is increased relative to equilibrium and the abundance of $\mathrm{NH}_{3}$ is decreased relative to equilibrium (e.g., Saumon et al. 2003, 2006). The presence of this $\mathrm{NH}_{3}$ band also makes it difficult to assess whether they exhibit the $1.015 \mu \mathrm{m} \mathrm{CH}_{4}$ band that we tentatively identified in the data in Section 3.

The derived $T_{\text {eff }}$ and $\log g$ values, $350 \mathrm{~K} / 5.0\left(\mathrm{~cm} \mathrm{~s}^{-2}\right)$ and $400 \mathrm{~K} / 4.5\left(\mathrm{~cm} \mathrm{~s}^{-2}\right.$ ), for the Morley et al. (solar composition, partly cloudy) and Sonora Bobcat models (cloud-free, with varied metallicity and $\mathrm{C} / \mathrm{O}$ ), respectively, are similar. The corresponding age of $26.7 \mathrm{Gyr}$ for the Morley et al. model (a result of the high surface gravity of $\log g=5$ ) is clearly inconsistent with the age of the universe. This underscores the fact that care must be taken when deriving the atmospheric properties of $\mathrm{Y}$ dwarfs because while atmospheric models can be constructed with various effective temperatures and surface gravities, objects with these atmospheres may not actually exist in our universe (see also Schneider et al. 2015). The Sonora Bobcat model gives a more realistic age of $3.14 \mathrm{Gyr}$, but either has a large disparity between the $d_{C}$ values of $17.9 \mathrm{pc}$ and the known distance of $9.93 \pm 0.23 \mathrm{pc}$ or an unphysical $R_{C}$ of $0.56 \mathcal{R}_{\mathrm{J}}^{\mathrm{N}}$.

Turning to the fits of the full spectral energy distribution shown in the bottom row of Figure 7, we see that while both models generally reproduce the very red near-to-mid-infrared color of WISE 1828+2650, the Morley et al. (2014) models fail to reproduce the [3.6] point while the Sonora Bobcat models fail to reproduce the $W 2$ and [4.5] points. The $T_{\text {eff }}$ and $\log g$ values of $275 \mathrm{~K} / 4.5\left(\mathrm{~cm} \mathrm{~s}^{-2}\right)$ for the Morley et al. model fit result in model bolometric luminosity and $d_{\mathrm{C}}$ values that are completely inconsistent with observations and a large $R_{\mathrm{C}}$ that would require an unrealistically young age of a few megayears at these temperatures. The Sonora Bobcat model matches the observed luminosity and distance better, which indicates that if WISE $1828+2650$ is a single object, it has a mass of $\sim 5 \mathcal{M}_{\text {Jup }}$, an age of $760 \mathrm{Myr}$, and both a subsolar metallicity and subsolar $\mathrm{C} / \mathrm{O}$ ratio.

The mismatch at [3.6] between the data and the models that assume solar abundances is a well-known problem (e.g., Leggett et al. 2017). Thus, the much better agreement between the [3.6] point and the Sonora Bobcat models is most likely a result of the fact that the Sonora models have $[\mathrm{M} / \mathrm{H}]$ and $[\mathrm{C} / \mathrm{O}]$ as free parameters, since the best-fitting model has $[\mathrm{M} / \mathrm{H}]=-0.5$ and $[\mathrm{C} / \mathrm{O}]=-0.6$. Figure 10 shows a sequence of model spectra with typical $\mathrm{Y}$ dwarf $T_{\text {eff }}$ and $g$ values but with variations in $[\mathrm{M} / \mathrm{H}]$ and $[\mathrm{C} / \mathrm{O}]$. Changes in both metallicity and $[\mathrm{C} / \mathrm{O}]$ affect the width of the flux peak at $4.5 \mu \mathrm{m}$ in the sense that lower metallicity and lower [C/O] values result in a widening of the blue side of the emission peak. While both the Spitzer [3.6] and [4.5] bands sample this side of the peak, so little emission emerges from the model brown dwarf in the short-wavelength half of the [3.6] band that small changes in the emission peak result in a large change in 

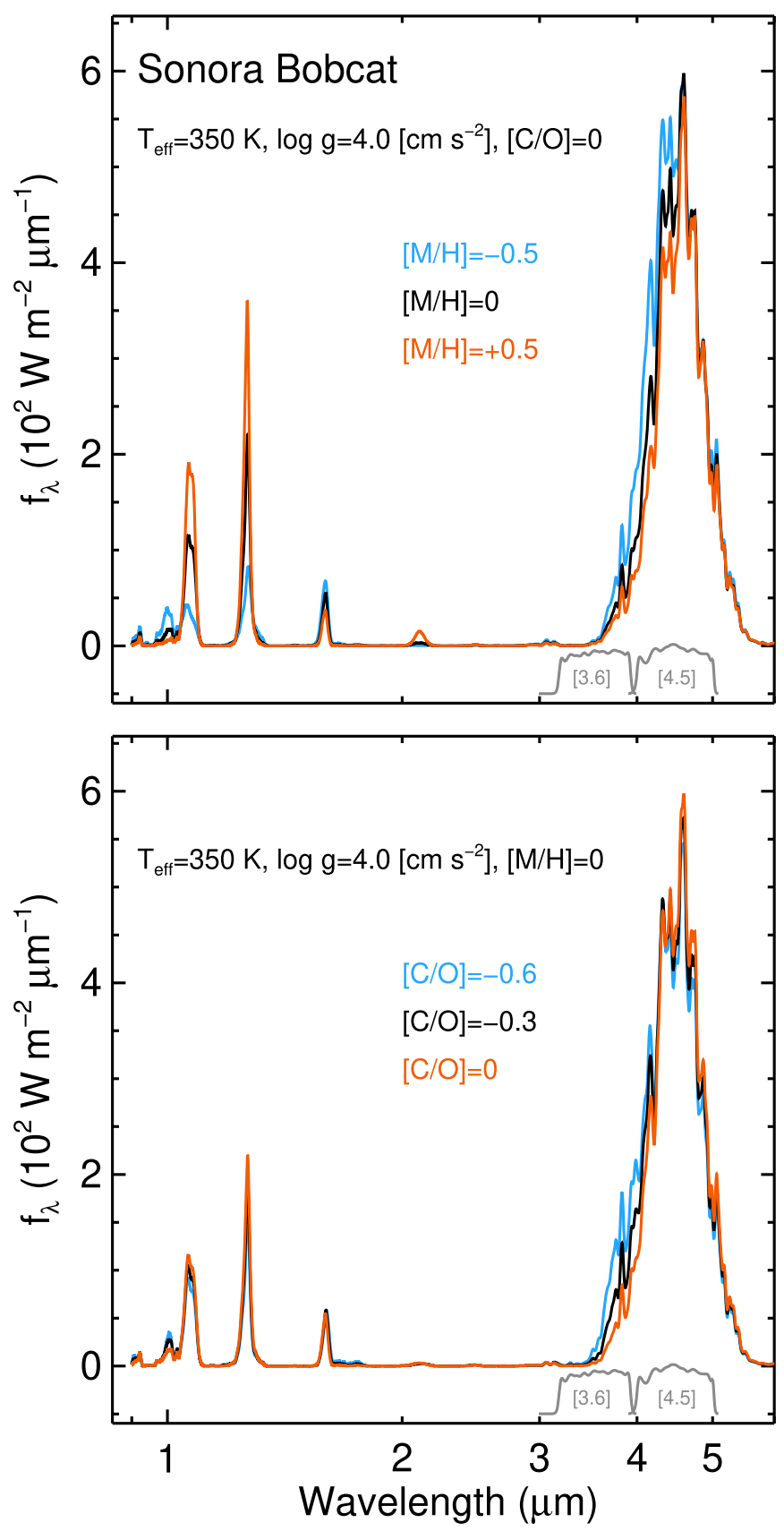

Figure 10. Sonora model spectra with atmospheric parameters typical for a $\mathrm{Y}$ dwarf $\left(T_{\text {eff }}=350 \mathrm{~K}, \log g=4.0\left(\mathrm{~cm} \mathrm{~s}^{-2}\right)\right.$ when $[\mathrm{M} / \mathrm{H}]$ and $[\mathrm{C} / \mathrm{O}]$ are varied. The models have been smoothed to $R \approx 180$ and correspond to the emergent flux at the top of the atmosphere. Also shown in gray are the transmission curves for the Spitzer/IRAC [3.6] and [4.5] bands.

the [3.6] magnitude. Previous work by Morley et al. (2018) and Zalesky et al. (2019) has also shown that subsolar [C/O] values are required in order to match the observations of $\mathrm{Y}$ dwarfs. The aforementioned vertical mixing also impacts the carbon chemistry and thus the abundances of $\mathrm{CO}$ and $\mathrm{CH}_{4}$. Since the opacity of these molecules sculpts the $5 \mu \mathrm{m}$ flux peak, nonequilibrium carbon chemistry can also have an impact on how well the Spitzer and WISE points are reproduced by the models (e.g., Hubeny \& Burrows 2007; Miles et al. 2020). Including both nonsolar $\mathrm{C} / \mathrm{O}$ ratios and nonequilibrium carbon chemistry in forward-model fits to $\mathrm{Y}$ dwarf observations may therefore be required in order to accurately reproduce the spectral morphology of the $5 \mu \mathrm{m}$ flux peak.
Finally, we also plot the best-fitting models to the HST spectrum over the best-fitting models to the spectral energy distribution and vice versa. The differences between the fits at near-infrared wavelengths is minimal, but the HST spectrum fit fails to reproduce the dramatic rise in flux at $\sim 5 \mu \mathrm{m}$. This underscores the difficulty of estimating the atmospheric properties of $\mathrm{Y}$ dwarfs with data sets that have limited wavelength coverage (see also Schneider et al. 2015).

The fits to the binary models are shown in Figure 8. With the exception of the Sonora Bobcat fit to the entire spectral energy distribution (lower right panel), the binary models provide identical fits to those of the single models (the $d_{C}$ values of these models are a factor of $\sqrt{2}$ larger than the single fits and remain a poor match to the observed distance of $9.93 \mathrm{pc}$ ). However, the Sonora Bobcat binary model fits the $5 \mu \mathrm{m}$ peak much better than a single model, and as a result, the $\chi^{2}$ falls from a value of 7838 to 7286 . Perhaps most importantly, $d_{\mathrm{C}}=9.03 \mathrm{pc}$, which is reasonably close to the measured value of 9.93. As a result, a rough but consistent picture between the data and the atmospheric and evolutionary models emerges whereby WISE $1828+2650$ is a $\sim 1$ Gyr old binary composed of two $T_{\text {eff }} \sim 325 \mathrm{~K}, \sim 5 M_{\text {Jup }}$ brown dwarfs with subsolar $[\mathrm{C} / \mathrm{O}]$ ratios.

This research is based on observations made with the NASA/ESA Hubble Space Telescope obtained from the Space Telescope Science Institute, which is operated by the Association of Universities for Research in Astronomy, Inc., under NASA contract NAS 526555. These observations are associated with program 12970 . This publication makes use of data products from the Wide-field Infrared Survey Explorer, which is a joint project of the University of California, Los Angeles, and the Jet Propulsion Laboratory/California Institute of Technology, and NEOWISE, which is a project of the Jet Propulsion Laboratory/California Institute of Technology. WISE and NEOWISE are funded by the National Aeronautics and Space Administration. This research has benefited from the Y Dwarf Compendium maintained by Michael Cushing at https://sites.google.com/view/ydwarfcompendium/.

Facilities: HST (WFC3), Spitzer (IRAC).

Software: IDL.

\section{ORCID iDs}

Michael C. Cushing (i) https://orcid.org/0000-0001-7780-3352 Adam C. Schneider (i) https://orcid.org/0000-0002-6294-5937 J. Davy Kirkpatrick (D) https://orcid.org/0000-00034269-260X

Caroline V. Morley (iD https://orcid.org/0000-0002-4404-0456

Mark S. Marley (1) https://orcid.org/0000-0002-5251-2943

Gregory N. Mace (1) https://orcid.org/0000-0001-7875-6391

Edward L. Wright (iD https://orcid.org/0000-0001-5058-1593

Michael F. Skrutskie (iD https://orcid.org/0000-0001-

8671-5901

Kenneth A. Marsh (iD https://orcid.org/0000-0003-0107-7803

\section{References}

Ackerman, A. S., \& Marley, M. S. 2001, ApJ, 556, 872

Bardalez Gagliuffi, D. C., Faherty, J. K., Schneider, A. C., et al. 2020, ApJ, 895,145

Beichman, C., Gelino, C. R., Kirkpatrick, J. D., et al. 2013, ApJ, 764, 101

Bochanski, J. J., Burgasser, A. J., Simcoe, R. A., \& West, A. A. 2011, AJ, 142,169 
Bowles, N., Calcutt, S., Irwin, P., \& Temple, J. 2008, Icar, 196, 612

Burgasser, A. J., Geballe, T. R., Leggett, S. K., Kirkpatrick, J. D., \& Golimowski, D. A. 2006, ApJ, 637, 1067

Burgasser, A. J., Kirkpatrick, J. D., Liebert, J., \& Burrows, A. 2003, ApJ, 594, 510

Burningham, B., Pinfield, D. J., Leggett, S. K., et al. 2008, MNRAS, 391, 320

Burrows, A., Hubbard, W. B., Lunine, J. I., \& Liebert, J. 2001, RvMP, 73, 719

Burrows, A., Sudarsky, D., \& Lunine, J. I. 2003, ApJ, 596, 587

Cruikshank, D. P., \& Binder, A. B. 1969, CoLPL, 6, 275

Cushing, M. C. 2014, in 50 Years of Brown Dwarfs, ed. V. Joergens (Cham: Springer)

Cushing, M. C., Kirkpatrick, J. D., Gelino, C. R., et al. 2011, ApJ, 743, 50

Cushing, M. C., Marley, M. S., Saumon, D., et al. 2008, ApJ, 678, 1372

Cushing, M. C., Roellig, T. L., Marley, M. S., et al. 2006, ApJ, 648, 614

Cutri, R. M., Wright, E. L., Conrow, T., et al. 2013, Explanatory Supplement to the AllWISE Data Release Products, Tech. Rep.

Dahn, C. C., Harris, H. C., Vrba, F. J., et al. 2002, AJ, 124, 1170

Delorme, P., Albert, L., Forveille, T., et al. 2010, A\&A, 518, A39

Delorme, P., Delfosse, X., Albert, L., et al. 2008a, A\&A, 482, 961

Delorme, P., Willott, C. J., Forveille, T., et al. 2008b, A\&A, 484, 469

Dupuy, T. J., \& Kraus, A. L. 2013, Sci, 341, 1492

Faherty, J. K., Tinney, C. G., Skemer, A., \& Monson, A. J. 2014, ApJL, 793, L16

Filippazzo, J. C., Rice, E. L., Faherty, J., et al. 2015, ApJ, 810, 158

Hubeny, I., \& Burrows, A. 2007, ApJ, 669, 1248

Irwin, P. G. J., Calcutt, S. B., Sihra, K., et al. 1999, JQSRT, 62, 193

Irwin, P. G. J., Teanby, N. A., \& Davis, G. R. 2010, Icar, 208, 913

Jarrett, T. H., Cohen, M., Masci, F., et al. 2011, ApJ, 735, 112

Kimble, R. A., MacKenty, J. W., O'Connell, R. W., \& Townsend, J. A. 2008 Proc. SPIE, 7010, 70101E

Kirkpatrick, J. D. 2008, in ASP Conf. Ser. 384, 14th Cambridge Workshop on Cool Stars, Stellar Systems, and the Sun, ed. G. van Belle (San Francisco, CA: ASP), 85

Kirkpatrick, J. D., Cushing, M. C., Gelino, C. R., et al. 2011, ApJS, 197, 19 Kirkpatrick, J. D., Gelino, C. R., Cushing, M. C., et al. 2012, ApJ, 753, 156 Kirkpatrick, J. D., Gelino, C. R., Faherty, J. K., et al. 2021, ApJS, 253, 7

Kirkpatrick, J. D., Martin, E. C., Smart, R. L., et al. 2019, ApJS, 240, 19

Kuntschner, H., Kümmel, M., Walsh, J. R., \& Bushouse, H. 2011, Revised Flux Calibration of the WFC3 G102 and G141 grisms, ST-ECF Instrument Science Rep., WFC3-2011-05

Lawrence, A., Warren, S. J., Almaini, O., et al. 2007, MNRAS, 379, 1599

Leggett, S. K., Marley, M. S., Freedman, R., et al. 2007, ApJ, 667, 537

Leggett, S. K., Morley, C. V., Marley, M. S., et al. 2013, ApJ, 763, 130

Leggett, S. K., Morley, C. V., Marley, M. S., \& Saumon, D. 2015, ApJ, 799, 37

Leggett, S. K., Tremblin, P., Esplin, T. L., Luhman, K. L., \& Morley, C. V. 2017, ApJ, 842, 118
Liu, M. C., Delorme, P., Dupuy, T. J., et al. 2011, ApJ, 740, 108

Lodders, K., \& Fegley, B. 2002, Icar, 155, 393

Lodieu, N., Béjar, V. J. S., \& Rebolo, R. 2013, A\&A, 550, L2

Luhman, K. L. 2014, ApJL, 786, L18

Luhman, K. L., Burgasser, A. J., \& Bochanski, J. J. 2011, ApJL, 730, L9

Luhman, K. L., \& Esplin, T. L. 2016, AJ, 152, 78

Mamajek, E. E., Prsa, A., Torres, G., et al. 2015, arXiv:1510.07674

Marley, M., \& Saumon, D. 2020, Sonora 2018: Cloud-free, Solar C/O Substellar Evolution and Photometry, nc_m+0.0_co1.0_v1.0, Zenodo, doi: 10.5281 /zenodo. 3733843

Marley, M., Saumon, D., Morley, C., \& Fortney, J. 2018, Sonora 2018: Cloudfree, Solar Composition, Solar C/O Substellar Atmosphere Models and Spectra, nc_m+0.0_co1.0_v1.0, Zenodo, doi: 10.5281/zenodo.1309035

Marley, M. S., \& Leggett, S. K. 2009, in The Future of Ultracool Dwarf Science with JWST, ed. H. A. Thronson, M. Stiavelli, \& A. Tielens (Dordrecht: Springer), 101

Marley, M. S., Saumon, D., \& Goldblatt, C. 2010, ApJL, 723, L117

Marley, M. S., Saumon, D., \& Visscher, C. 2021, ApJ, accepted (arXiv:2107. 07434)

Marocco, F., Caselden, D., Meisner, A. M., et al. 2019, ApJ, 881, 17

Marocco, F., Eisenhardt, P. R. M., Fowler, J. W., et al. 2021, ApJS, 253, 8

Marocco, F., Kirkpatrick, J. D., Meisner, A. M., et al. 2020, ApJL, 888, L19

Meisner, A. M., Caselden, D., Kirkpatrick, J. D., et al. 2020, ApJ, 889, 74

Miles, B. E., Skemer, A. J. I., Morley, C. V., et al. 2020, AJ, 160, 63

Morley, C. V., Marley, M. S., Fortney, J. J., et al. 2014, ApJ, 787, 78

Morley, C. V., Skemer, A. J., Allers, K. N., et al. 2018, ApJ, 858, 97

Rayner, J. T., Cushing, M. C., \& Vacca, W. D. 2009, ApJS, 185, 289

Reach, W. T., Megeath, S. T., Cohen, M., et al. 2005, PASP, 117, 978

Roellig, T. L., Van Cleve, J. E., Sloan, G. C., et al. 2004, ApJS, 154, 418

Saumon, D., Geballe, T. R., Leggett, S. K., et al. 2000, ApJ, 541, 374

Saumon, D., Marley, M. S., Abel, M., Frommhold, L., \& Freedman, R. S. 2012, ApJ, 750, 74

Saumon, D., Marley, M. S., Cushing, M. C., et al. 2006, ApJ, 647, 552

Saumon, D., Marley, M. S., Lodders, K., \& Freedman, R. S. 2003, in IAU Symp. 211, Brown Dwarfs, ed. E. Martín (San Francisco, CA: ASP), 345

Schneider, A. C., Cushing, M. C., Kirkpatrick, J. D., et al. 2015, ApJ, 804, 92

Schneider, A. C., Cushing, M. C., Kirkpatrick, J. D., \& Gelino, C. R. 2016, ApJL, 823, L35

Stephens, D. C., Leggett, S. K., Cushing, M. C., et al. 2009, ApJ, 702, 154

Tinney, C. G., Faherty, J. K., Kirkpatrick, J. D., et al. 2014, ApJ, 796, 39

Tokunaga, A. T., \& Vacca, W. D. 2005, PASP, 117, 421

Wright, E. L., Eisenhardt, P. R. M., Mainzer, A. K., et al. 2010, AJ, 140, 1868

Yurchenko, S. N., Barber, R. J., \& Tennyson, J. 2011, MNRAS, 413, 1828

Yurchenko, S. N., \& Tennyson, J. 2014, MNRAS, 440, 1649

Zalesky, J. A., Line, M. R., Schneider, A. C., \& Patience, J. 2019, ApJ, 877, 24 